\title{
GLOBAL DYNAMICS OF SUBSURFACE SOLAR ACTIVE REGIONS
}

\author{
L. Jouve ${ }^{1,2}$, A. S. Brun ${ }^{2,4}$, ANd G. Aulanier ${ }^{3}$ \\ ${ }^{1}$ UPS-OMP, Institut de Recherche en Astrophysique et Planétologie, Université de Toulouse CNRS, \\ 14 Avenue Edouard Belin, F-31400 Toulouse, France; ljouve@irap.omp.eu \\ ${ }^{2}$ Laboratoire AIM, CEA/DSM-CNRS-Université Paris Diderot, IRFU/SAp, F-91191 Gif sur Yvette, France \\ ${ }^{3}$ LESIA, Observatoire de Paris, CNRS, UPMC, Université Paris-Diderot, 5 Place Jules Janssen, F-92190 Meudon Cedex, France \\ Received 2012 June 29; accepted 2012 October 31; published 2012 December 7
}

\begin{abstract}
We present three-dimensional numerical simulations of a magnetic loop evolving in either a convectively stable or unstable rotating shell. The magnetic loop is introduced into the shell in such a way that it is buoyant only in a certain portion in longitude, thus creating an $\Omega$-loop. Due to the action of magnetic buoyancy, the loop rises and develops asymmetries between its leading and following legs, creating emerging bipolar regions whose characteristics are similar to those of observed spots at the solar surface. In particular, we self-consistently reproduce the creation of tongues around the spot polarities, which can be strongly affected by convection. We further emphasize the presence of ring-shaped magnetic structures around our simulated emerging regions, which we call "magnetic necklace" and which were seen in a number of observations without being reported as of today. We show that those necklaces are markers of vorticity generation at the periphery and below the rising magnetic loop. We also find that the asymmetry between the two legs of the loop is crucially dependent on the initial magnetic field strength. The tilt angle of the emerging regions is also studied in the stable and unstable cases and seems to be affected both by the convective motions and the presence of a differential rotation in the convective cases.
\end{abstract}

Key words: convection - magnetic fields - magnetohydrodynamics (MHD) - methods: numerical Sun: interior - Sun: rotation

Online-only material: color figures

\section{INTRODUCTION}

Our turbulent active Sun possesses a rather regular magnetic cycle that has been observed directly through the photospheric emergence of sunspots for about four centuries now. This magnetic flux emergence, which occurs daily at the Sun's surface, appears not only at large scales (sunspots and active regions) but also at scales as small as the intra-network field and there is indication that even smaller scale flux emerges at the solar surface, which remains unresolved with the present magnetographs (Sanchez Almeida 1998; van Driel-Gesztelyi 2002). However, since a huge amount of flux is brought toward the photosphere by solar active regions and because of the wellestablished robust properties of such large-scale emergence, we focus our studies on the understanding of the dynamical evolution of large and strong magnetic structures rising from the solar interior to the surface. The appearance of active regions can be summarized in a few steps: small magnetic bipoles first emerge all over the future active region and then merge to form sunspots at the outer edges of the emerging flux region (EFR). The growing phase of a typical EFR lasts about three to five days. The flux in an active region is usually larger than $10^{21} \mathrm{Mx}$ in each polarity. The orientation of the bipole may be arbitrary at first, but generally in one to three days it becomes slightly tilted $\left(3^{\circ}-10^{\circ}\right)$ with respect to the east-west direction, with the leading polarity closer to the equator, thus conforming to Joy's law (Hale et al. 1919). The tilt angles of individual active regions show a large scatter about the mean but the measurements indicate a tendency for the mean value to linearly increase with the latitude of emergence (Wang \& Sheeley 1989, 1991; D'silva $\&$ Choudhuri 1993). Moreover, various asymmetries have been

\footnotetext{
4 Associated to LESIA, Observatoire de Paris, CNRS, UPMC, Université
} Paris-Diderot, 5 Place Jules Janssen, F-92190 Meudon Cedex, France. detected in EFRs, in particular in the morphology of the leading and trailing spots. Indeed, the leading polarity generally appears more intense and concentrated than the trailing one which tends to be more fragmented. Another interesting feature of EFRs is their inherent twist. Indeed, careful multi-wavelength analysis of flux emergence in active regions (e.g., Leka et al. 1996) indicates that they appear with a certain amount of twist (i.e., a non-zero current along their axis). This twist is then thought to be responsible for the characteristic pattern known as tongues observed in newly emerging regions (Lopez Fuentes et al. 2000) and identified as a major ingredient in mass ejections or flares at the solar photosphere (e.g., Fan \& Gibson 2004; Török \& Kliem 2005; Aulanier et al. 2010). Other structures, such as an annular shape of the magnetic field around active regions, have been observed through various instruments (e.g., Liu \& Zhang 2006) but never really reported as identified structures. However, as we will see, they can serve as strong constraints on models and simulations, and we will point out the relevant physical processes responsible for their existence.

With all of those observational constraints in mind, theoreticians and modelers have tried to analyze in detail the dynamical evolution of strong magnetic structures from the base of the convection zone $(\mathrm{CZ})$ where they are created to the surface where they appear as sunspots. A possible scenario for such an evolution would be that strong toroidal fields are created at the base of the CZ by the differential rotation shearing a preexisting poloidal field in the tachocline (see landmark papers of Moffatt 1978 and Parker 1993, recent simulations of Browning et al. 2006, and a review of the solar dynamo theory by Ossendrijver 2003 for example). After a certain period of storage in the subadiabatic layer below the CZ (Moreno-Insertis et al. 1992; Schüssler et al. 1994), this toroidal field becomes subject to magnetic buoyancy instabilities (Parker 1955; Acheson 1979; Cattaneo \& Hughes 
1988; Matthews et al. 1995) and organizes itself in buoyant arched magnetic flux tubes (or $\Omega$-loops; Zwann 1987). The rise of those loops under the influence of Coriolis forces, turbulent convection, and large-scale flows (rotation and meridional circulation) would then produce the wide variety of large-scale flux emergence observed at the solar surface, with the robust properties summarized above. Several numerical experiments of this last step have been performed using various levels of approximation, from the classical "thin flux tube" models (Spruit 1981; Choudhuri \& Gilman 1987) to the more recent two-dimensional and three-dimensional calculations (see Fan 2004 for a detailed review on this subject). In particular, Emonet \& Moreno-Insertis (1998) showed the necessity of starting with a twisted magnetic field to counteract the vorticity generation within the flux tube and its subsequent breakup. Fan et al. (2003) demonstrated the significant effect of convective motions on the deformation of the magnetic loop when the initial field was comparable to the equipartition field strength (corresponding to a magnetic energy equal to the kinetic energy of the strongest downflows). More recently, Jouve \& Brun (2009) considered the effects of spherical geometry, convective motions, and self-consistently developed mean flows (differential rotation and meridional flow) on a rising flux tube. It was found that an initial twist of the field lines of about 14 turns along the $360^{\circ}$ longitude as well as an initial field strength of about $130 \mathrm{kG}$ were necessary for a coherent radial rise of the tube, that the tilt angle was significantly influenced not only by the initial twist and Coriolis force but also by the local convection, and that meridional circulation was able to advect initially weak flux tubes toward the poles when they reached the top of the domain. They showed, moreover, a quantitative study of the effect of magnetic diffusivity by varying the Prandtl number in their simulations. Fan (2008) also investigated the effects of spherical geometry, but on an $\Omega$-loop (a flux tube made buoyant in a limited range of longitudes), and thus focused on the properties of simulated individual active regions, but without convection. Here, we propose to compute the dynamical evolution of an $\Omega$-loop both in an isentropic and fully convective spherical shell, seeking to answer the following questions.

1. What are the physical processes at the origin of the observed tilt angles of active regions and is it possible to reproduce Joy's law?

2. What is the influence of convective motions on a rising $\Omega$-loop and the subsequent bipolar emerging regions?

3. Can we quantify the effects of large-scale mean flows?

4. How important is the twist of the magnetic structures in the evolution within the convection zone and on the morphology of EFRs?

5. How does the structure of simulated active regions compare to observations?

This work is thus an attempt to answer those questions using numerical simulations of a convective spherical shell, using the anelastic spherical harmonic (ASH) code which solves the MHD equations under the anelastic approximation, valid within the bulk of the convection zone. We do not address here the evolution of emerging flux in the solar atmosphere, which needs to be computed in a fully compressible simulation. Extensive studies of magnetic flux emergence in the atmosphere have been performed (e.g., Magara 2004; Manchester et al. 2004; Murray et al. 2006; Martínez-Sykora et al. 2008; Archontis \& Török 2008) which showed that magnetic flux reaching the photosphere could undergo a dynamical expansion into the atmosphere as a result of the nonlinear growth of the magnetic buoyancy instability and that it is difficult for a twisted flux tube to rise bodily into a corona because of the heavy plasma trapped in the magnetic dips. Successive magnetic reconnections could, however, help to achieve actual emergence of twisted magnetic loops into the corona (Pariat et al. 2004).

This article is organized as follows. Section 2 presents the model chosen, the hydrodynamic background, as well as the initial conditions which will favor the emergence of individual active regions. The following short Section 3 presents the various cases calculated, which will be considered in an isentropic background (without convection) and with convective motions acting on the magnetic loop. Sections 4 and 5, respectively, present the results of the MHD simulations in the isentropic and convective cases. Finally, we discuss the results and conclude in Section 6.

\section{THE MODEL}

\subsection{Anelastic MHD Equations}

All of the calculations presented in this work were computed with the ASH code, which solves the anelastic magnetohydrodynamic equations in a rotating spherical shell. ASH uses a pseudo-spectral method in space and a semi-implicit approach in time (e.g., Clune et al. 1999; Miesch et al. 2000; Brun et al. 2004). The full nonlinear evolution of the velocity and magnetic fields is computed, whereas the equations are linearized in thermodynamic variables with respect to a spherically symmetric mean state to have density $\bar{\rho}$, pressure $\bar{P}$, temperature $\bar{T}$, and specific entropy $\bar{S}$. Perturbations are denoted as $\rho, P, T$, and $S$. The equations being solved are

$$
\begin{gathered}
\nabla \cdot(\bar{\rho} \mathbf{v})=0 \\
\nabla \cdot \mathbf{B}=0 \\
\bar{\rho}\left[\frac{\partial \mathbf{v}}{\partial t}+(\mathbf{v} \cdot \nabla) \mathbf{v}+2 \Omega_{0} \times \mathbf{v}\right]=-\nabla P+\rho \mathbf{g} \\
+\frac{1}{4 \pi}(\nabla \times \mathbf{B}) \times \mathbf{B}-\nabla \cdot \mathcal{D}-[\nabla \bar{P}-\bar{\rho} \mathbf{g}], \\
\bar{\rho} \bar{T} \frac{\partial S}{\partial t}+\bar{\rho} \bar{T} \mathbf{v} \cdot \nabla(\bar{S}+S)=\nabla \cdot\left[\kappa_{r} \bar{\rho} c_{p} \nabla(\bar{T}+T)\right. \\
+\kappa \bar{\rho} \bar{T} \nabla \bar{S}+\kappa \bar{\rho} \bar{T} \nabla S]+\frac{4 \pi \eta}{c^{2}} \mathbf{j}^{2} \\
+2 \bar{\rho} v\left[e_{i j} e_{i j}-\frac{1}{3}(\nabla \cdot \mathbf{v})^{2}\right] \\
\frac{\partial \mathbf{B}}{\partial t}=\nabla \times(\mathbf{v} \times \mathbf{B})-\nabla \times(\eta \nabla \times \mathbf{B}),
\end{gathered}
$$

where $\mathbf{v}=\left(v_{r}, v_{\theta}, v_{\phi}\right)$ is the local velocity in spherical coordinates in the frame rotating at a constant angular velocity $\Omega_{0}, \mathbf{g}$ is the gravitational acceleration, $\mathbf{B}=\left(B_{r}, B_{\theta}, B_{\phi}\right)$ is the magnetic field, $\mathbf{j}=(c / 4 \pi)(\nabla \times \mathbf{B})$ is the current density, $c_{p}$ is the specific heat at constant pressure, $\kappa_{r}$ is the radiative diffusivity (fitted to a one-dimensional seismically calibrated solar model; see Brun et al. 2002), $\eta$ is the effective magnetic diffusivity, and $\mathcal{D}$ is the viscous stress tensor. To represent the unresolved subgrid-scale processes, the code uses an effective 
eddy viscosity $v$ and an effective thermal eddy diffusivity. They are both here to model the enhanced diffusion associated with turbulent convective motions in the bulk of the convection zone. In the present simulations, they are chosen to vary in radius only as $\bar{\rho}^{-1 / 3}$. The thermal diffusion $\kappa_{0}$ acting on the mean entropy gradient (contrary to the thermal eddy diffusivity $\kappa$ which acts on the entropy perturbation) occupies a narrow region in the upper convection zone. Its purpose is to transport heat through the outer surface where radial convective motions vanish (Gilman \& Glatzmaier 1981; Wong \& Lilly 1994), and is thus chosen such that the entire luminosity will be transported at the top of the domain by the so-called unresolved heat flux $F_{u}=-\kappa_{0} \bar{\rho} \bar{T} d \bar{S} / d r$. The diffusivity $\kappa$ is purely dissipative and acts to smooth out entropy variations, whereas $\kappa_{0}$ is essentially a cooling term near the top of our computational domain. To complete the set of equations, we use the linearized equation of state,

$$
\frac{\rho}{\bar{\rho}}=\frac{P}{\bar{P}}-\frac{T}{\bar{T}}=\frac{P}{\gamma \bar{P}}-\frac{S}{c_{p}},
$$

where $\gamma$ is the adiabatic exponent (equal to $5 / 3$ here), and assume the ideal gas law

$$
\bar{P}=\mathcal{R} \bar{\rho} \bar{T}
$$

where $\mathcal{R}$ is the ideal gas constant, taking into account the mean molecular weight $\mu$ corresponding to a mixture composed roughly of $3 / 4$ of hydrogen and $1 / 4$ of helium per mass. The reference or mean state (indicated by overbars) is derived from a one-dimensional solar structure model (Brun et al. 2002). It begins in hydrostatic balance so that the bracketed term on the right-hand side of Equation (4) initially vanishes. However, as the simulation evolves, turbulent and magnetic pressures drive the reference state slightly away from strict hydrostatic balance.

The ASH code uses a spectral decomposition for all the variables, namely, spherical harmonics in the horizontal direction and Chebyshev polynomials in radius. The collocation points are thus the zeros of the Legendre polynomials in latitude, uniformly distributed in longitude, and the distribution is denser at both ends of the domain in the radial direction. The code integrates the MHD equations presented above over $0.72 R_{\odot} \leqslant r \leqslant 0.96 R_{\odot}$ (with an overall density contrast of about 24 between top and bottom), $0 \leqslant \theta \leqslant \pi$, and $0 \leqslant \phi \leqslant 2 \pi$. The resolution is chosen here such that at least 20 points in radius and in latitude are contained inside the flux tube section. The minimal resolution used here is thus $N_{r} \times N_{\theta} \times N_{\phi}=256 \times 512 \times 1024$.

Finally, the boundary conditions for the velocity are impenetrable and stress-free at the top and bottom of the shell. We impose a constant entropy gradient top and bottom for the isentropic case and for the fully convective case, a latitudinal entropy gradient is imposed at the bottom, as in Miesch et al. (2006). In all cases, we match the magnetic field to an external potential magnetic field at the top and bottom of the shell (Brun et al. 2004).

\subsection{Initial Conditions to Favor the Creation of $\Omega$-loops}

In this paper, we implement initial conditions for the magnetic field similar to what was used in Jouve \& Brun (2009). An initially axisymmetric magnetic structure is embedded in an unmagnetized stratified medium. In order to keep a divergenceless magnetic field, we use a toroidal-poloidal decomposition,

$$
\mathbf{B}=\nabla \times \nabla \times\left(C \mathbf{e}_{r}\right)+\nabla \times\left(A \mathbf{e}_{r}\right)
$$

the expressions used for the potentials $A$ and $C$ for the flux tubes are

$$
\begin{gathered}
A=-A_{0} r \exp \left[-\left(\frac{r-R_{t}}{a}\right)^{2}\right] \times\left[1+\tanh \left(2 \frac{\theta-\theta_{t}}{a / R_{t}}\right)\right] \\
C=-A_{0} \frac{a^{2}}{2} q \exp \left[-\left(\frac{r-R_{t}}{a}\right)^{2}\right] \times\left[1+\tanh \left(2 \frac{\theta-\theta_{t}}{a / R_{t}}\right)\right],
\end{gathered}
$$

where $A_{0}$ is a measure of the initial field strength, $a$ is the tube radius, $\left(R_{t}, \theta_{t}\right)$ is the position of the tube center, and $q$ is the twist parameter.

In Jouve \& Brun (2009), we derived an expression for the winding degree of the field lines (i.e., the number of turns that the field lines make over the whole tube length $2 \pi R_{t} \sin \theta_{t}$, assuming a uniform twist):

$$
n=\frac{\pi R_{t} \sin \theta_{t}}{2 a} \tan \psi
$$

where the tangent of the pitch angle $\psi$ is related to the twist parameter via the following expression, considering that we are at $\theta=\theta_{t}$ and at the tube periphery $r=R_{t}+a$, as in Jouve \& Brun (2009):

$$
\tan \psi \approx \frac{q a}{R_{t}+a}
$$

In all cases, the tube radius is set to $a=2 \times 10^{9} \mathrm{~cm}$, about a 10th of the depth of the modeled convection zone, and is introduced at the base of the $\mathrm{CZ}$ at $R_{t}=5.2 \times 10^{10} \mathrm{~cm}$. If we consider that the typical flux in an observed active region is $10^{21}-10^{22} \mathrm{Mx}$ and that an initial flux tube has a magnetic field of about $5 \times 10^{4} \mathrm{G}$ as in our simulations, then the conservation of flux tells us that the radius of the loop should in reality be closer to $8 \times 10^{7} \mathrm{~cm}$. Our flux tubes are thus bigger than what could be expected. Different values for the loop radius have been shown to influence flux emergence (D'silva \& Choudhuri 1993; Emonet \& Moreno-Insertis 1998; see also the discussion of Section 6). Here, we limit our parameter study to a fixed loop radius (see Jouve \& Brun 2009, for a different set of parameters). The initial field strength $A_{0}$, the initial twist of the field lines $q$, and the latitude of introduction $\theta_{t}$ will be varied in our models to investigate the influence of these various parameters.

In order to obtain a flux tube that is buoyant on a small portion in longitude only, we initially apply a perturbation on the background entropy field located at the initial position of the flux tube in $(r, \theta)$ and possessing a Gaussian profile in longitude $\phi$. The entropy perturbation thus has the following expression:

$$
\begin{aligned}
S_{\text {in }}-S_{\text {ext }}= & A_{S} \times \exp \left[-\left(\frac{r-R_{t}}{a}\right)^{2}\right] \\
& \times \frac{2 R_{t}}{a} \times \frac{1}{\cosh ^{2}\left(2 \frac{\theta-\theta_{t}}{a / R_{t}}\right)} \\
& \times\left[-C_{S}+\exp \left(-\left(\frac{\phi-\phi_{0}}{\phi_{e}}\right)^{2}\right)\right],
\end{aligned}
$$

where $A_{S}$ is the amplitude of the entropy perturbation, $C_{S}$ is a constant value controlling the buoyancy of the rest of the loop, $\phi_{0}$ is the longitude of maximum perturbation (or buoyancy), and $\phi_{e}$ is the extent of the entropy perturbation. 
The effect of such a perturbation on entropy is to produce an additional density deficit inside the flux tube at a particular location in longitude. As a consequence, we can derive the new maximum density contrast between the tube and its surroundings, which will result from this additional perturbation. Total pressure equilibrium is not enforced at the beginning of the simulation and since an initial magnetic tension is also present in most of our cases (the twisted cases), the loop is not initially in mechanical equilibrium outside the portion which is made buoyant, even if $C_{S}$ is modified. However, there is an initial adjustment visible at the very early stages of the dynamical evolution where the pressure perturbation inside the loop will tend to compensate for the magnetic pressure, in particular, the loop gets slightly squeezed in an Alfvén time at the very beginning of the simulation, without much influence on the following evolution. We thus derive here only an indication of the efficiency of the magnetic buoyancy in a situation of pressure equilibrium and entropy perturbation (not exactly satisfied here) by writing the following relations, respectively, for the total pressure and the entropy:

$$
\begin{gathered}
P_{\mathrm{ext}}^{g}-P_{\mathrm{in}}^{g}=\frac{B^{2}}{8 \pi} \\
\operatorname{Max}\left(S_{\mathrm{in}}-S_{\mathrm{ext}}\right)=\Delta S=A_{S} \times \frac{2 R_{t}}{a} \times\left(1-C_{S}\right),
\end{gathered}
$$

where $P_{\mathrm{ext}}^{g}$ and $P_{\mathrm{in}}^{g}$ are the gas pressure, respectively, outside and inside the tube and where the maximum of $S_{\text {in }}-S_{\text {ext }}$ is given by Equation (13) and taken at $r=R_{t}, \theta=\theta_{t}$, and $\phi=\phi_{0}$. We then use an additional relation to make the density $\rho$ appear:

$$
S=c_{v} \ln P-c_{p} \ln \rho,
$$

with $c_{p}$ the specific heat at constant pressure (equal here to $\left.3.4 \times 10^{8} \mathrm{erg} \mathrm{K} \mathrm{K}^{-1} \mathrm{~g}^{-1}\right), c_{v}$ the specific heat at constant volume, and $\gamma=c_{p} / c_{v}=5 / 3$ the adiabatic index.

These equalities lead to the following relation between entropy, pressure, and density inside and outside of the flux tube,

$$
\Delta S=\operatorname{Max}\left(S_{\mathrm{in}}-S_{\mathrm{ext}}\right)=\operatorname{Max}\left(c_{v} \ln \frac{P_{\mathrm{in}}}{P_{\mathrm{ext}}}-c_{p} \ln \frac{\rho_{\mathrm{in}}}{\rho_{\mathrm{ext}}}\right),
$$

and thus we find an expression for the maximum buoyancy of our magnetic structures:

$$
\begin{aligned}
\frac{\Delta \rho}{\rho}= & \operatorname{Max}\left(\frac{\rho_{\mathrm{ext}}-\rho_{\mathrm{in}}}{\rho_{\mathrm{ext}}}\right)=1-\exp \left(-\frac{\Delta S}{c_{p}}\right)\left(1-\frac{B_{0}^{2}}{8 \pi P_{\mathrm{ext}}}\right)^{1 / \gamma} \\
& \approx 1-\left(1-\frac{\Delta S}{c_{p}}\right)\left(1-\frac{B_{0}^{2}}{8 \pi \gamma P_{\mathrm{ext}}}\right),
\end{aligned}
$$

where $B_{0}$ will be the maximum field strength on the tube axis. A first-order Taylor expansion has been done to give an approximate value of the buoyancy, considering that both $\Delta S / c_{p}$ and $B_{0}^{2} / 8 \pi P_{\text {ext }}$ are equally small values. In the remainder of the paper, we will use the following parameters for the standard cases for the entropy perturbation: $C_{S}=0.35, \phi_{e}=15^{\circ}$, $\phi_{0}=100^{\circ}$, and the average external pressure used to estimate the maximum buoyancy will be $P_{\text {ext }}=4 \times 10^{13}$ dynes. We use a fixed value for $C_{S}$ since we do not seek to maintain a perfect mechanical equilibrium for the rest of the loop, otherwise the value of $C_{S}$ should depend on the magnetic field.

\subsection{The Background Hydrodynamical Models}

The same hydrodynamical background was used in this work as in Jouve \& Brun (2009). Namely, two situations will be investigated. In the first, the magnetic structure is introduced in an isentropic layer with solid body rotation and without convection. In the second situation, the convective instability is triggered and differential rotation as well as a large-scale meridional flow are self-consistently created within the bulk of the convection zone. The competition between buoyant loops and convective motions and large-scale flows in this case will be studied and compared to the stably stratified situation. In particular, we focus on the shape of the developing loop, its asymmetries, and the morphology of the emerging radial field it generates. Moreover, in this study, we focus on the comparison to observations of large-scale EFRs, extrapolating the magnetic configurations we get from our simulations at the upper boundary (i.e., $0.96 R_{\odot}$ ) to what could happen higher up, closer to the photosphere. We specifically investigate the evolution of flux, tilt angle, and spot morphology during emergence.

\section{CASES CALCULATED}

In this study, we investigate seven different cases. All of the parameters used for each case are summarized in Table 1. Four of the cases come as pairs with the same buoyancy (but different field strength and entropy perturbation: Cases 1 \& 3 and 2 \& 4) and the last three are extreme cases (the weakest Cases 0a and $\mathrm{Ob}$ and strongest field Case 5). For Cases 1, 2, 3, and 4, the twist parameter was also varied. The cases with a right-handed (or Positive) twist (corresponding to $q=30$ ) will be referred to by the case number with the suffix TwP, and for the left-handed (or Negative) twist, we will add TwN. For example, the case where $B_{0}=5 \times 10^{4} \mathrm{G}, \Delta S=3380$ (corresponding to a buoyancy $\Delta \rho / \rho$ of $1.1 \times 10^{-5}$ according to Equation (18)), and which has a right-handed twist $q=30$ will be called Case 1TwP.

Some additional cases were also computed:

Case 1 with the loop introduced at a latitude of $60^{\circ}$; Case 1 TwP60 and $15^{\circ}$; Case 1TwP15.

Case 3 without twist; Case 3NoTw and with the twist at its threshold value (defined in the last paragraph of Section 4.1.1); Case 3TwTh.

The values of the entropy perturbation were chosen to ensure a rise time of the same order of the convective turnover time, i.e., between 10 and 40 days approximately. We can compare the buoyancy of the loops with and without the entropy perturbation by setting $\Delta S=0$ in Equation (18). We find that the value of $\Delta \rho / \rho$ is multiplied by 7.65 when the entropy perturbation is introduced in Case 1 (compared to the case without entropy perturbation) and by a factor 1.41 in Case 5 , so that the stronger field cases are less influenced by the entropy perturbation applied. Moreover, the values of the entropy perturbations used here are comparable in amplitude to the typical fluctuations measured at the base of our computational domain in our convective runs.

\section{EVOLUTION OF A SINGLE LOOP WITHOUT CONVECTION}

We first compute the evolution of a single $\Omega$-loop evolving in a stable background with respect to convection. It will first confirm that we indeed correctly control the buoyancy of the 
Table 1

Key Parameters of the Various Cases

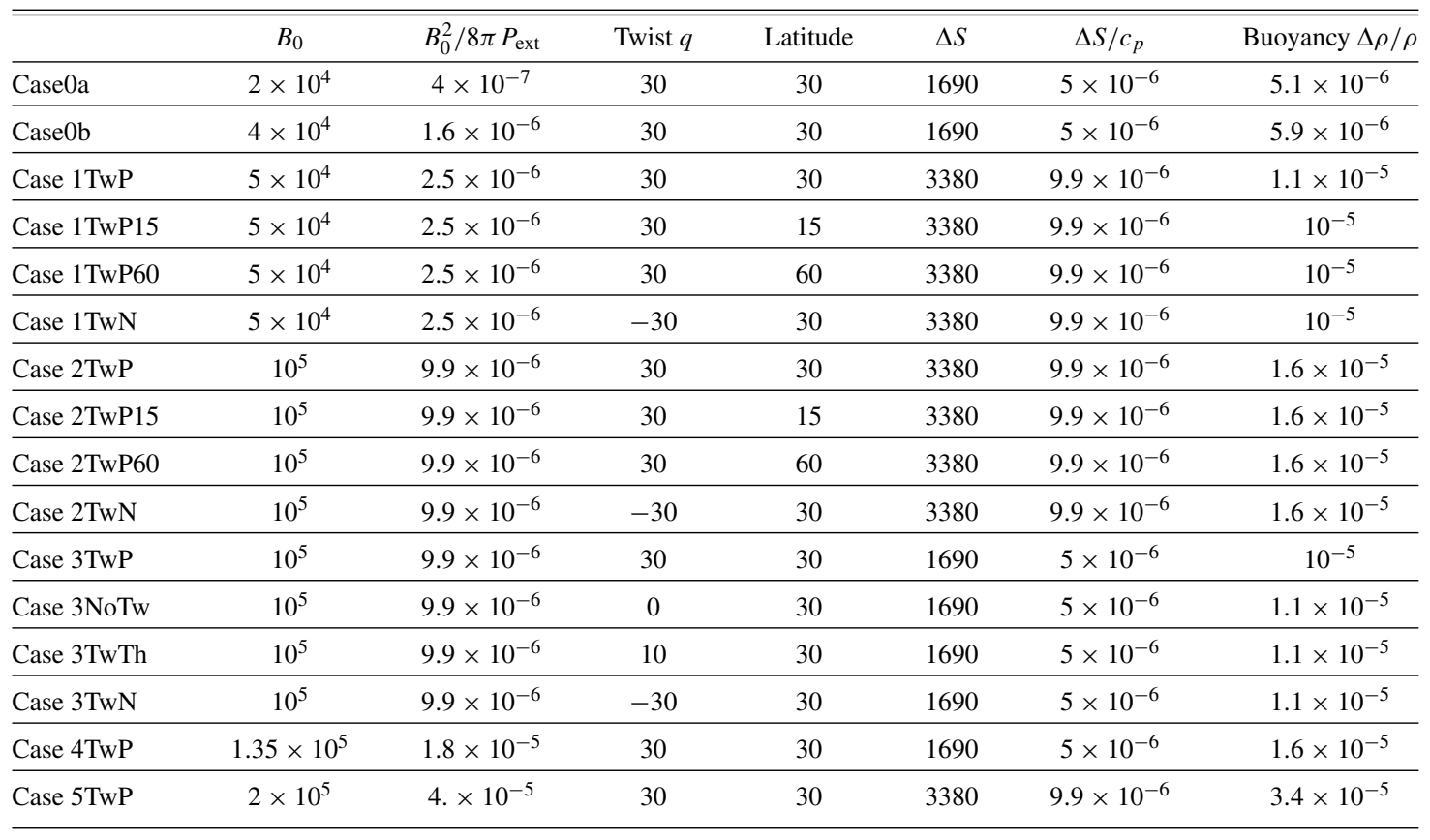

Notes. The amplitude of the magnetic field is given in $\mathrm{G}$, latitude in degrees, and entropy perturbation $\Delta S$ in erg $\mathrm{K}^{-1} \mathrm{~g}^{-1}$.

flux tube with the initial conditions we apply. Second, it will help us see if the important parameters for the evolution of a uniformly buoyant flux tube are still the same here and what differences may be pointed out.

\subsection{Favorable Conditions for Emergence}

\subsubsection{Twist}

We know from previous experiments that a certain amount of twist is needed for a magnetic flux tube to be able to rise coherently in a convectively stable (or unstable) background (e.g., Emonet \& Moreno-Insertis 1998). Indeed, in a non-twisted case, the azimuthal vorticity created by the gravitational torque acting on the loop cannot be compensated. Two counter vortices thus quickly appear and break the loop apart, preventing it from emerging at the surface. It was argued previously that this situation may differ when an $\Omega$-loop is considered, instead of a uniformly buoyant structure (Abbett et al. 2000). The additional magnetic tension coming from the deformation of the magnetic torus may oppose the vorticity generation.

Figure 1 shows the section of an untwisted $\Omega$-loop corresponding to Case 3 NoTw of Table 1 . The zoom is made on the longitude where the loop becomes buoyant, thanks to the combined effects of magnetic pressure and entropy perturbation. Two instants in the evolution are shown. Here, we represent the contours of the azimuthal (or axial) component of the magnetic field superimposed on the azimuthal vorticity. It is rather clear in this case (especially on the left panel) that strong vortices are formed inside the flux tube, which have the tendency to significantly deform the magnetic structure. However, as the loop rises further up, it becomes more arched and more magnetic tension is generated. As a consequence, even without any twist, some coherence is maintained in this case, even if a large amount of flux is lost in the process.

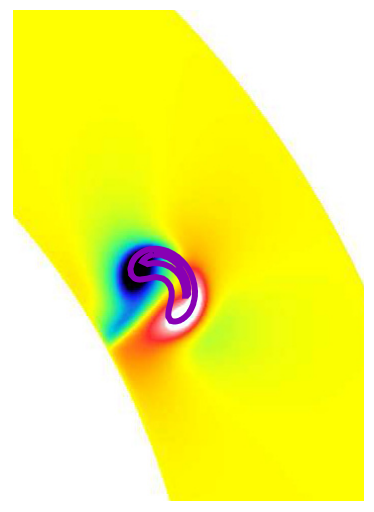

Time $=5$ days

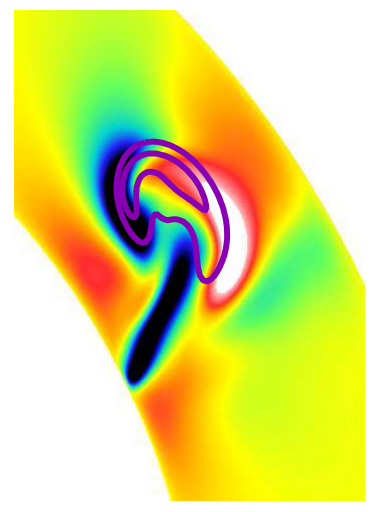

Time $=13.6$ days
Figure 1. Cross section of the untwisted $\Omega$-loop of Case 3NoTw, introduced at a latitude of $30^{\circ}$, at two instants in the evolution, 5 and 13.6 days. Magenta contours of the azimuthal component of the magnetic field are superimposed to the azimuthal vorticity which presents a characteristic profile of two counter vortices.

(A color version of this figure is available in the online journal.)

This flux loss is visible in Figure 2 where the profile of the toroidal magnetic field is shown at the radius where it peaks after about six days of evolution, as a function of colatitude. As a reminder, the flux ropes were introduced here at $60^{\circ}$ of colatitude and with an amplitude of $10^{5} \mathrm{G}$. In the nontwisted case (dashed line on the figure), we clearly see that the two counter vortices have created two distinct magnetic flux concentrations, whose maximum amplitude reaches a value of $2.5 \times 10^{4} \mathrm{G}$ while the maximum amplitude of the twisted case is $4 \times 10^{4} \mathrm{G}$. The flux rope has been eroded by the vorticity generation and the two vortices tend to dominate the further evolution of the magnetic structure, which separates into two flux concentrations. However, along the loop (following its axis), this double-peak structure is not as clearly visible as at the 


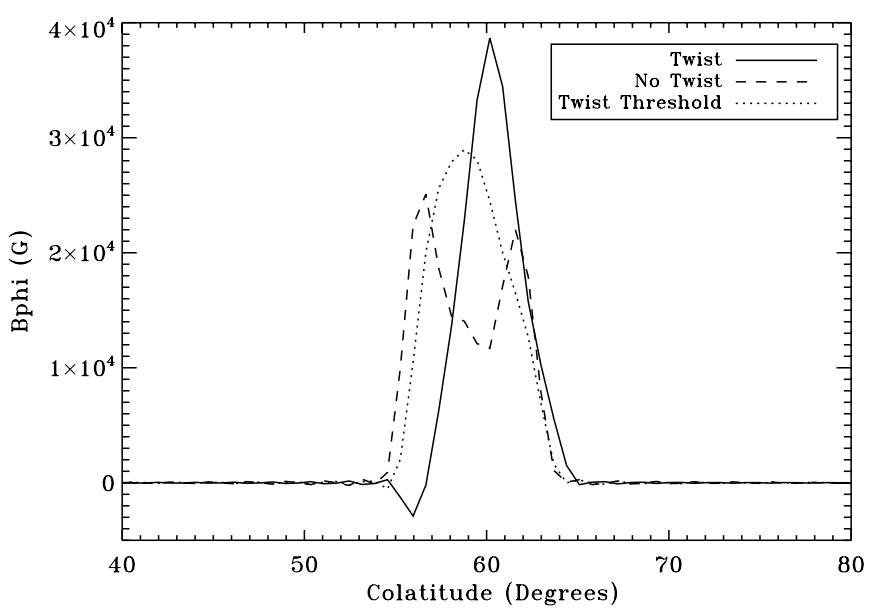

Figure 2. Profile of the toroidal field at the radius where it peaks after 6 days of evolution (i.e., $0.82 R_{\odot}$ ). The three cases represented are the untwisted case $(q=0)$ Case 3NoTw, the twisted case Case 3TwP $(q=30)$, and the intermediate case Case 3TwTh where the twist value is at its threshold $(q=10)$.

longitude we chose here. This feature may be the consequence of the asymmetry which develops between the leading and trailing legs. We will come back to this in the next section.

We decided to define the twist threshold as the value above which the double-peak structure is no longer present at any longitude. With this criterion, we find that the twist threshold for this case is $q=10$, corresponding to 0.54 turns along the $15^{\circ}$ of extension of the $\Omega$-loop. We note that this threshold value is reduced by a factor 1.8 compared to the uniformly buoyant flux tubes of Jouve \& Brun (2009). This is due to the additional tension coming from the arching of the $\Omega$-loop in this case, which already has the effect of limiting the vorticity generation inside the magnetic structure. This result is in agreement with the work of Abbett et al. (2000). The case where the twist is set at its threshold value is also represented in Figure 2 (dotted line). In this case, the maximum value of the field is reduced compared to the more twisted case but the coherence of the loop is kept.

\subsubsection{Buoyancy versus Rotation}

The buoyancy force is proportional to $\Delta \rho$, which is given by expression (18). While the loop rises because of the buoyancy force, a retrograde longitudinal flow develops in order to conserve angular momentum (see section below). As a consequence, a Coriolis force oriented toward the rotation axis and perpendicular to this axis also develops, opposing the component of the buoyancy force perpendicular to the rotation axis. The only uncompensated force is then parallel to the rotation axis and oriented toward the pole. If the buoyancy force is not strong enough, then the loop will have the tendency to rise parallel to the rotation axis and emerge at a much higher latitude than where it was introduced. This is the case at fixed $\Delta S$ when the magnetic energy is not strong enough. We recover this situation when the poloidal field is too weak (corresponding to a low twist value, enough to maintain coherence of the loop but not enough to make it rise radially).

Figure 3 is an illustration of a case where the rise is mainly parallel to the rotation axis. In this Case $0 \mathrm{a}$, the twist was set to $q=30$ but the initial axial field strength was set to $2 \times 10^{4} \mathrm{G}$, and the entropy perturbation to the rather weak value of $\Delta_{S}=1680$, corresponding to a density perturbation of $\Delta \rho / \rho=5 \times 10^{-6}$. In this case, after 15 days of evolution, the trajectory of the loop

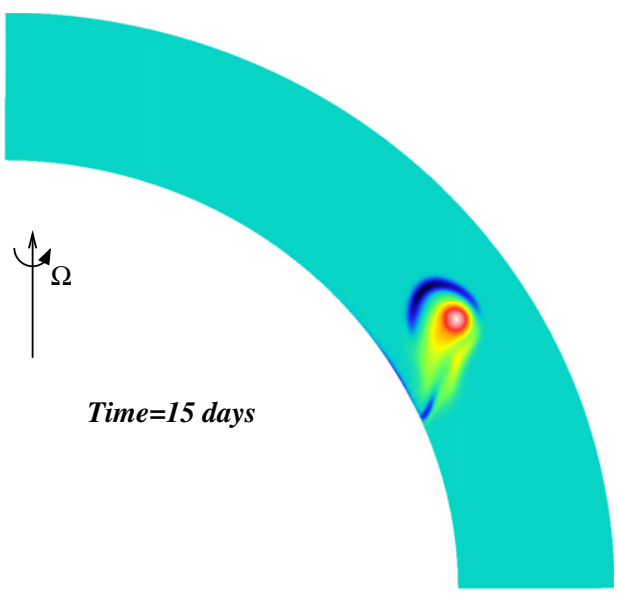

Figure 3. Section of $B_{\phi}$ at $t=15$ days at the longitude of maximum buoyancy for Case 0a. The trajectory of the loop is here clearly parallel to the rotation axis.

(A color version of this figure is available in the online journal.)

is clearly parallel to the rotation axis, since the Coriolis force has acted strongly on the magnetic field. Indeed, as shown by Choudhuri \& Gilman (1987) in the framework of the thin flux tube approximation, while the loop rises, it develops a retrograde longitudinal flow in its interior. A Coriolis force is associated with this longitudinal flow which tends to oppose the outward component of the buoyancy force perpendicular to the rotation axis, leaving only the component parallel to the rotation axis to act on the ring. Interestingly, Choudhuri \& Gilman (1987) find that in the adiabatic cases (similar to what we are studying in this section), the transition between vertical and radial rise happens between $\Delta \rho / \rho=2 \times 10^{-6}$ and $\Delta \rho / \rho=2 \times 10^{-5}$. We also find here that Cases $0 \mathrm{a}$ and $0 \mathrm{~b}$, where $\Delta \rho / \rho$ is around $5-6 \times 10^{-6}$, tend to be strongly influenced by the Coriolis force, while the stronger cases, where $\Delta \rho / \rho \geqslant 10^{-5}$, exhibit a much more radial trajectory.

We note that due to the low value of the buoyancy used for Case 0a, the rise of the loop is rather slow (about 40 days are needed to reach the top of our domain). In order to ensure an evolution dominated by the dynamical effects and not only by diffusion, we reduced the magnetic diffusivity from $7.95 \times 10^{11} \mathrm{~cm}^{2} \mathrm{~s}^{-1}$ to $1.59 \times 10^{11}$, and thus used a magnetic Prandtl of 5 instead of 1 as with all the other cases. It does not change the fact that the rise is parallel to the rotation axis in this case, it is just to compute a case where the rise time is significantly lower than the diffusive time (which would be of the order of $R^{2} / \eta=58$ days for the $P m=1$ case and thus too close to the rise time of 40 days).

The same kind of evolution parallel to the axis of rotation is found when stronger tubes are introduced with a lower twist. Indeed, if the twist is low as we saw before, then the amplitude of the magnetic field inside the loop quickly decreases until it reaches a value where the buoyancy force is not strong enough to keep the radial trajectory. This is visible on the right panel of Figure 1, where the initial axial field was $10^{5} \mathrm{G}$ but the initial poloidal field was 0 . To correctly define a minimum value for a radial rise here, it is thus necessary to take into account the three components of the magnetic field rather than focus on the axial field. Let us consider a case where the entropy perturbation is the weakest, corresponding to a slow rise and 


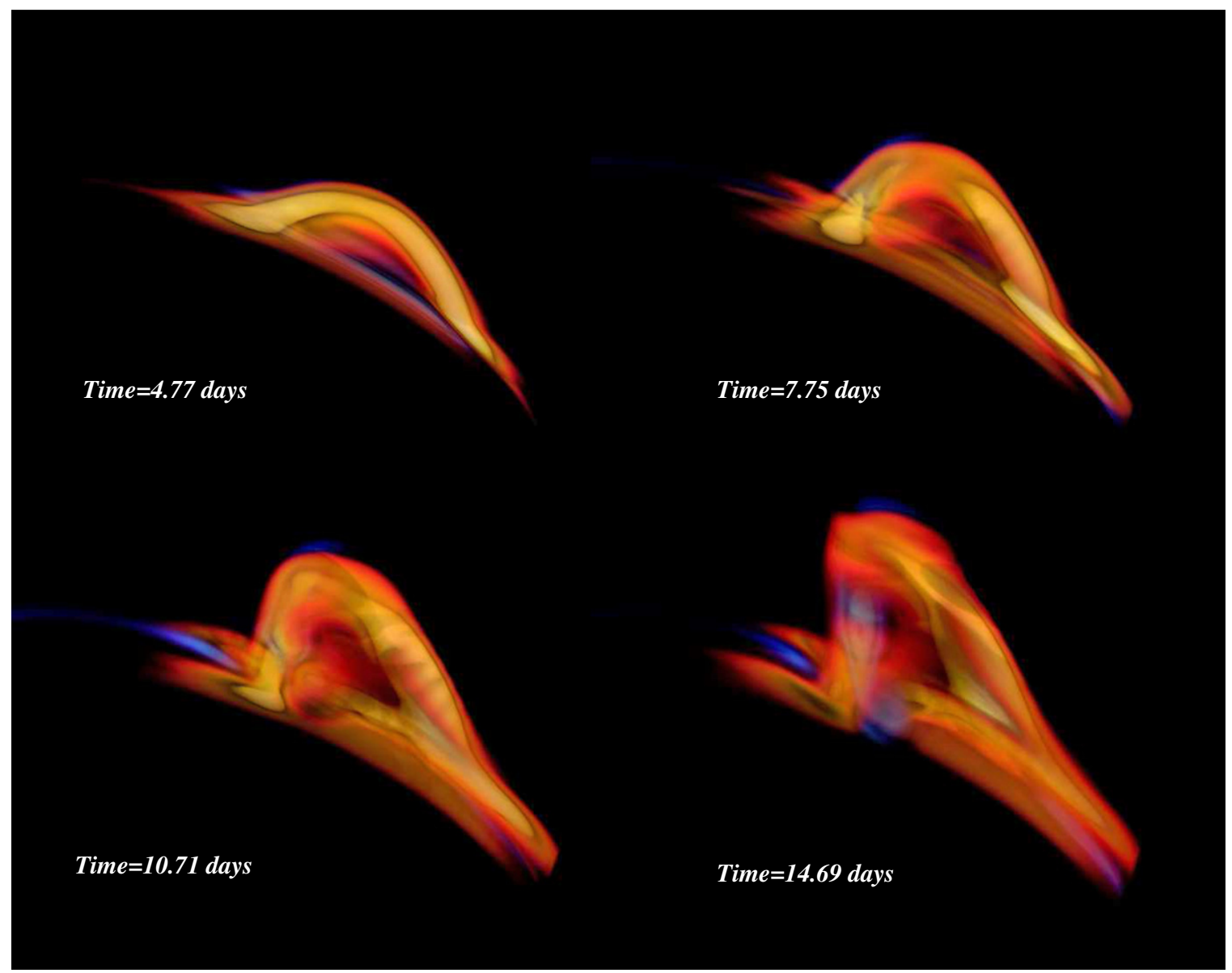

Figure 4. Volume rendering of $B_{\phi}$ while the loop rises through the isentropic layer in Case 1TwP. The loop is viewed up to the polar axis, from the South Pole. The asymmetry between the legs of the loop are obvious in these snapshots. The visualization was performed with SDvision@ cea (Pomarède \& Brun 2010).

(A color version of this figure is available in the online journal.)

then to a maximum effect of the Coriolis force. We then find that for a twist parameter $q=30$ (about one turn over the extension chosen here), the initial magnetic field needed for a radial rise should be at least $5 \times 10^{4} \mathrm{G}$. It can be argued that this value is strong compared to what could be expected at the base of the convection zone from dynamo action but it could be reduced if a stronger entropy perturbation was considered, which is locally possible in the turbulent environment existing at the base of the convection zone.

To summarize the two previous subsections, we can say that in order to maintain a coherent radial rise of our $\Omega$-loop in our simulation, an initial twist of 0.54 turns along the extension of the loop as well as an initial field strength of $50 \mathrm{kG}$ are necessary. We note that those two values are reduced compared to the uniformly buoyant calculations. This is promising since the observed twist values are of the order of less than one turn across the active region (Chae \& Moon 2005; Démoulin et al. 2002) and typical fields of more than $50 \mathrm{kG}$ are difficult to produce in present dynamo calculations (Brun et al. 2004).

\subsection{Asymmetry between the Trailing and Leading Legs}

We now study the evolution of a sufficiently twisted and strong loop so that it will maintain its coherence while it rises radially. The loop is introduced at $30^{\circ}$ in latitude, with a maximum buoyancy (i.e., entropy perturbation) located at $100^{\circ}$ in longitude, with a twist parameter $q=30$ representing about 39 turns around the tube axis over the whole $360^{\circ}$, and thus corresponding to about 1.6 turns over the extension of the buoyant part $\left(\phi_{e}=15^{\circ}\right)$. With this twist parameter (as we saw before), the loop rises radially for a field strength of $5 \times 10^{4} \mathrm{G}$. Figure 4 shows a volume rendering of the toroidal field evolution in Case 1TwP.

The asymmetry between the trailing and leading legs develops during the evolution and is clearly seen in the last two panels of the figure, at $t=10.7$ days and $t=14.7$ days. This section will be devoted to the study of this asymmetry and the influence of buoyancy and field strength. The action of rotation and the associated Coriolis force over the whole loop will also be detailed here. This process will lead to the appearance of a tilt of the axis with respect to the east-west direction and will also depend on the magnetic field strength, as we will see in the following section.

Let us enter now into the details of the asymmetry between the trailing and leading legs and the effect that the field strength has on it. This asymmetry, already seen in thin flux tube calculations (see Caligari et al. 1995), is due to the difference in azimuthal velocity between the top of the loop and its feet. Indeed, since the flux tube needs to conserve its angular momentum $r \sin \theta\left(r \sin \theta \Omega+v_{\phi}\right)$, a retrograde flow with respect to the rotating frame will develop inside the loop while it rises (i.e., while $r$ increases). Since the apex of the loop is higher than the feet, which stay attached to the bottom of the convection zone, a stronger retrograde flow appears at the apex. The profile of the flow along the magnetic structure then leads to the inclination asymmetry of the trailing and leading legs. In some of our 

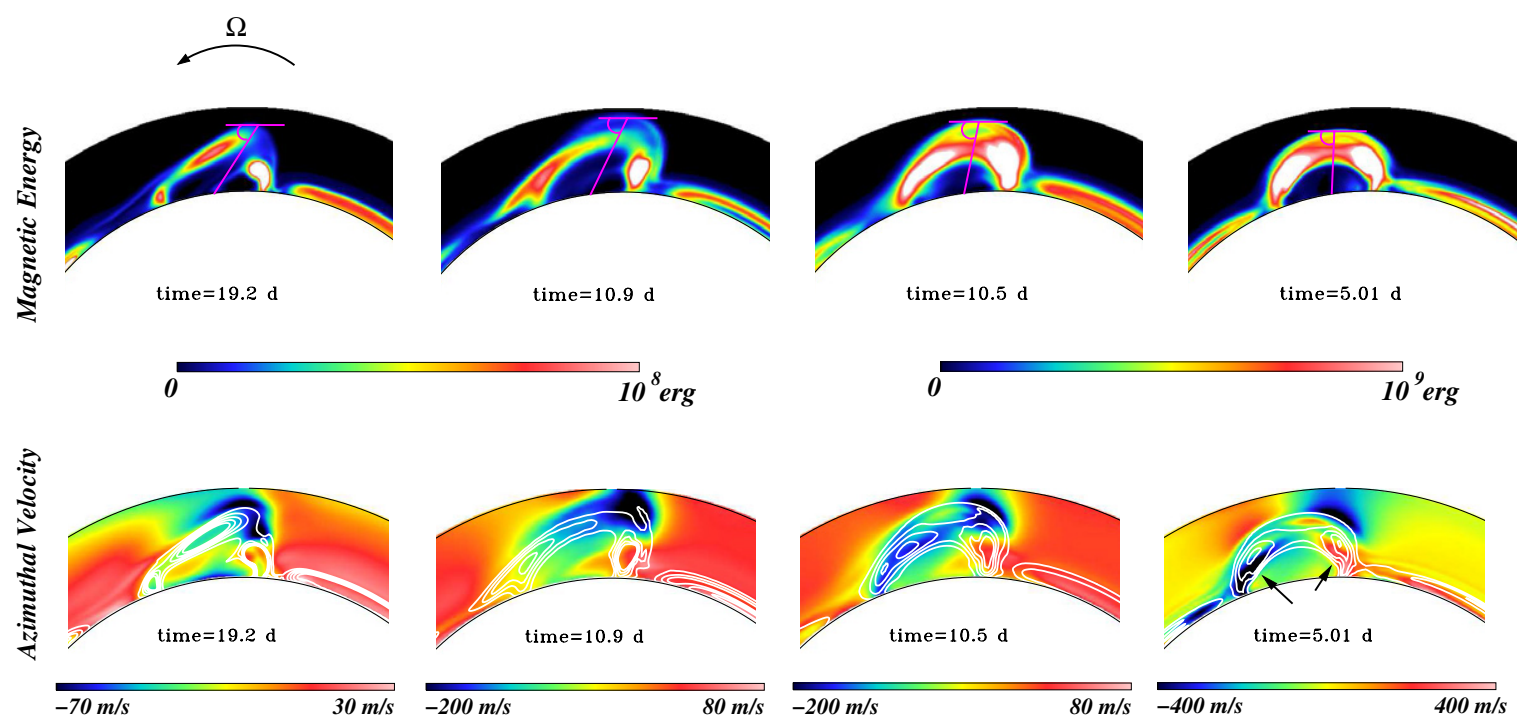

Figure 5. Cut at the latitude of $30^{\circ}$ of the magnetic energy (upper panel) and azimuthal velocity together with the white contours of magnetic energy (lower panel) for four different cases, Cases $0 \mathrm{~b}, 1 \mathrm{TwP}, 2 \mathrm{TwP}$, and 5TwP, when the $\Omega$-loops have approximately reached the same height $r=0.93 R_{\odot}$. The loops are viewed downward from the North Pole. The color scale used for the magnetic energy is the same for the first two panels and the same for the last two.

(A color version of this figure is available in the online journal.)

cases, since the parameter $C_{S}$ is always kept the same, parts of the loop located outside the buoyant portion can be slightly denser than their surroundings. As a consequence, those regions may sink toward the base of the convection zone and favor the difference in azimuthal velocity causing the asymmetry. However, in most cases (except Cases 0a, 0b, 1TwP, and 1TwN), the magnetic buoyancy can overcome this effect and the rest of the loop is slightly positively buoyant, thus instead reducing the asymmetry.

However, this explanation is valid as long as the Lorentz forces do not dominate the flow evolution inside the loop. Figure 5 shows a snapshot of the magnetic energy and the azimuthal velocity for four different cases: Cases 0b, 1TwP, 2TwP, and 5TwP. We note here that Case 0b has a magnetic field strength of $4 \times 10^{4} \mathrm{G}$ and is thus still significantly deflected toward the pole, but is an interesting case because it maximizes the asymmetry between the legs of the loop. The snapshots were chosen so that all loops have reached the same height (namely $0.93 R_{\odot}$ ), which corresponds to different physical times since the buoyancy force (i.e., the $\Delta \rho$ ) is different in all cases. In order to quantify the asymmetry, we measure the angle between the tangent at the apex and the line going through the middle of the two footpoints and the apex. Those lines are shown in magenta in the top panels of Figure 5. We find that the angles have the following values: $45^{\circ}, 50^{\circ}, 75^{\circ}$, and almost $90^{\circ}$ (meaning no asymmetry in this Case 5TwP, where the magnetic field and the buoyancy force are the strongest). We thus note that the asymmetry strongly decreases from Case 1TwP to Case 2TwP, even if the density deficits are initially not very different. The reason for this jump in the asymmetry is the difference in field strength $\left(10^{5} \mathrm{G}\right.$ for Case 2TwP against $5 \times 10^{4} \mathrm{G}$ for Case 1TwP). Indeed, in this case, the Lorentz force starts to become important to the generation of azimuthal velocity: strong values of $v_{\phi}$ appear at the location of strong gradients of the magnetic field. This is much more obvious in the stronger Case 5TwP. The profile of the azimuthal velocity shown on the last snapshot of the lower panel is clearly related to the gradients of the magnetic field, and the retrograde flow due to the conservation of angular momentum is so weak compared to the magnetic effects that it is not visible anymore. Instead, an azimuthal velocity directed toward the bisector of the tube appears on both sides of the loop (indicated by black arrows in the last panel), resulting in a symmetric arched structure (visible in the top right snapshot) with both feet showing an almost vertical orientation.

If we write down the expression of the Lorentz force involved in the evolution of $v_{\phi}$, we have

$$
\begin{aligned}
\frac{D v_{\phi}}{D t} & =\frac{J_{r} B_{\theta}-J_{\theta} B_{r}}{4 \pi}+\text { non-magnetic terms } \\
& =\frac{B_{\theta}}{r \sin \theta}\left(\frac{\partial}{\partial \theta}\left(\sin \theta B_{\phi}\right)-\frac{\partial B_{\theta}}{\partial \phi}\right) \\
& -\frac{B_{r}}{r}\left(\frac{1}{\sin \theta} \frac{\partial B_{r}}{\partial \phi}-\frac{\partial}{\partial r}\left(r B_{\phi}\right)\right)+\text { non-magnetic terms. }
\end{aligned}
$$

This equation shows that the regions of strong magnetic field gradients will correspond to the regions of production of $v_{\phi}$, which can be verified from the last panel of Figure 5, where the concentrations of $v_{\phi}$ are located mainly at the footpoints (where the gradients of $B_{r}$ and $B_{\theta}$ with respect to $\phi$ are strong) and higher up at the loop periphery (where the gradients of $B_{\phi}$ with respect to $r$ and $\theta$ are strong).

To be more precise, we can follow the evolution of the maximum and minimum of the azimuthal velocity which develops during the rise of the flux tube from the base of the convection zone toward the surface. In Figure 6, we plot the maximum and the minimum of $v_{\phi}$, in order to understand the differences in the asymmetries seen in Figure 5. For each case, the maximum and minimum azimuthal velocities are measured over the whole domain but those extreme values are always reached inside or in the vicinity of the magnetic structures. Moreover, the various cases (except for Case 0b) only differ by the initial magnetic field strength of the loop, and the differences in $v_{\phi}$ will thus be directly related to the differences in the magnetic properties. The most striking feature is the strong increase of the maximum velocity in all cases. This sharp increase happens in all cases when the loop reaches the mid-CZ, i.e., when the loop starts to curve significantly. If we look at Equation (19), we can understand that this sharp increase is due to the gradients with respect 

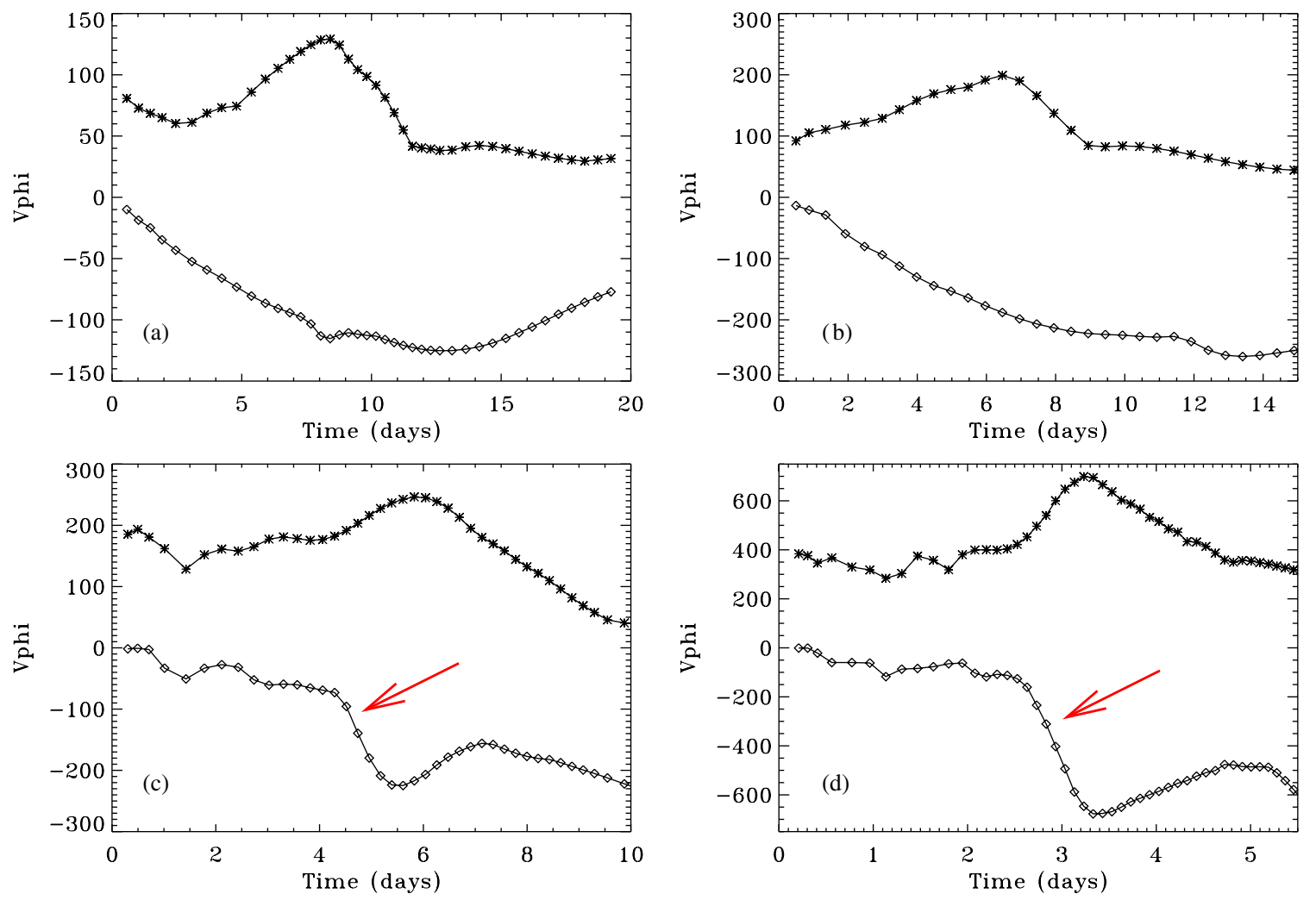

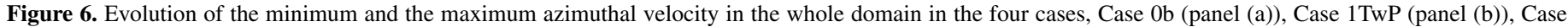
2TwP (panel (c)), and Case 5TwP (panel (d)), of Figure 5.

(A color version of this figure is available in the online journal.)

to the $\phi$-coordinate of $B_{r}$ and $B_{\theta}$, which only appear when the loop becomes arched, i.e., after a certain rise time, contrary to the gradients of $B_{\phi}$ with respect to $r$ and $\theta$, which already exist at the beginning of the simulation. If we now turn to look at the minimum of $v_{\phi}$, then we realize that this change of slope is also visible at the same time but only for the strong-field cases. They are indicated by the red arrows in panels (c) and (d). For the retrograde flow with respect to the rotating frame, another factor plays a role: the conservation of angular momentum. As we said before, it will have a tendency to produce a negative $v_{\phi}$ that is dominant in the weak-field cases (panels (a) and (b)) but dominated by the Lorentz force terms in the strong-field cases (panels (c) and (d)). As a consequence, strong gradients of the magnetic field and the associated magnetic tension will have the tendency to oppose the deformation of the loop while it rises, which explains why the asymmetry between the two legs of the loop is reduced when the initial field is strong enough (more than $10^{5} \mathrm{G}$ ). This feature was already observed in thin flux tube calculations (e.g., Moreno-Insertis et al. 1994; Caligari et al. 1995) and a similar argument involving the stiffness of intense fields was used to explain the weaker asymmetry seen in strongfield cases. We note, moreover, that since the rise time of strong tubes is smaller, the Coriolis force will have less time to act on the loop and the asymmetry will also be reduced because of shorter rise times.

Going back to Figure 4, we see that when the loop forms and rises, some negative toroidal field is created (blue colors in the figures), while the initial toroidal field was purely positive. This can be explained by a local $\Omega$-effect: the azimuthal velocity gradient built at the periphery of the loop while it rises will shear the small poloidal field component present at the tube boundary mainly. This $\Omega$-effect, related to a shearing of the poloidal field, is one of the main factors of the dynamo mechanism thought to be responsible for the generation of the magnetic field in differentially rotating astrophysical objects (see, for example, Brun 2004; Charbonneau 2005; Miesch et al. 2005 for a review on these processes in the solar context). The presence of such an effect could be surprising, especially in the untwisted case without convection, since no poloidal field or azimuthal velocity gradients exist at the initial stage. However, while the loop forms, the magnetic field is no longer purely toroidal since the field lines get curved and will thus get a mainly radial component. At the same time, gradients of azimuthal velocity will be created as shown above. Both of these effects are then favorable to obtain an $\Omega$-effect that will be able to create an additional toroidal field of both signs.

\subsection{Structure of the Emerging Regions}

We now turn to investigate the characteristics of the individual bipolar regions emerging at the top of our computational domain in all situations. In particular, we focus on the tilt angle, the asymmetry in the field strength in the two spots, and the morphological properties of the regions which can be related to observations.

\subsubsection{Evolution of the Tilt Angle: Effects of Twist and Latitude}

Here, we decide to focus on four cases: Cases 1TwP and 1TwN and Cases 3TwP and 3TwN. The main difference between the two cases with right-handed twist is the field strength which is multiplied by 2 in Case 3, but buoyancy is kept the same through a change of initial entropy perturbation. Moreover, we 
Positive Twist (right handed)
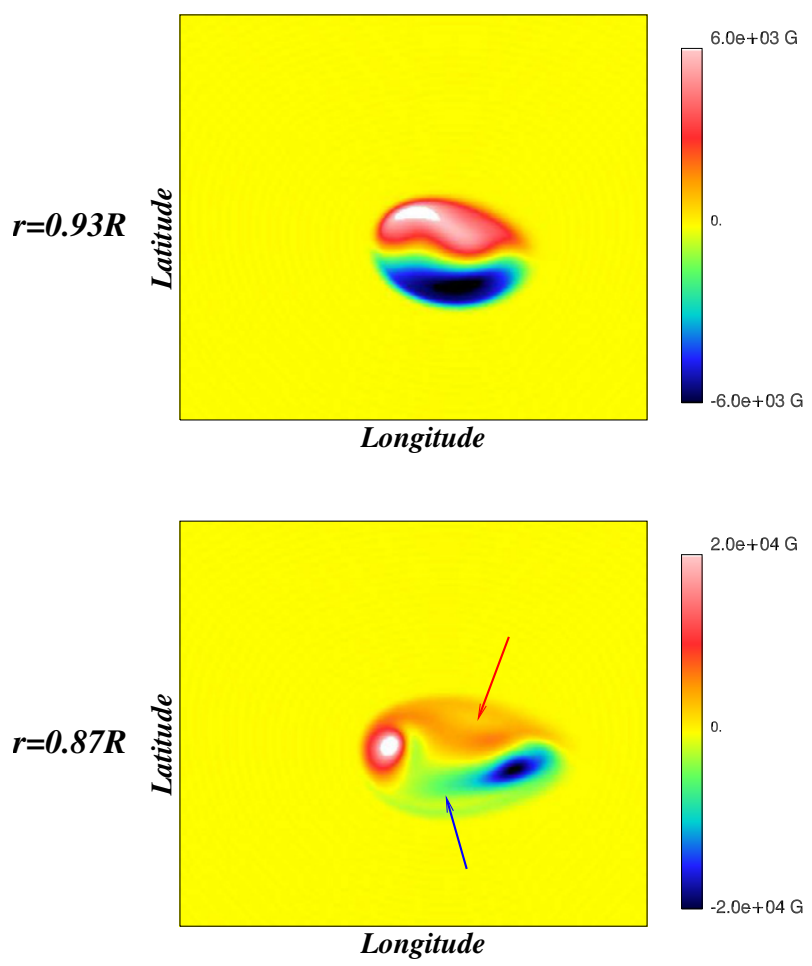

Negative Twist (left handed)
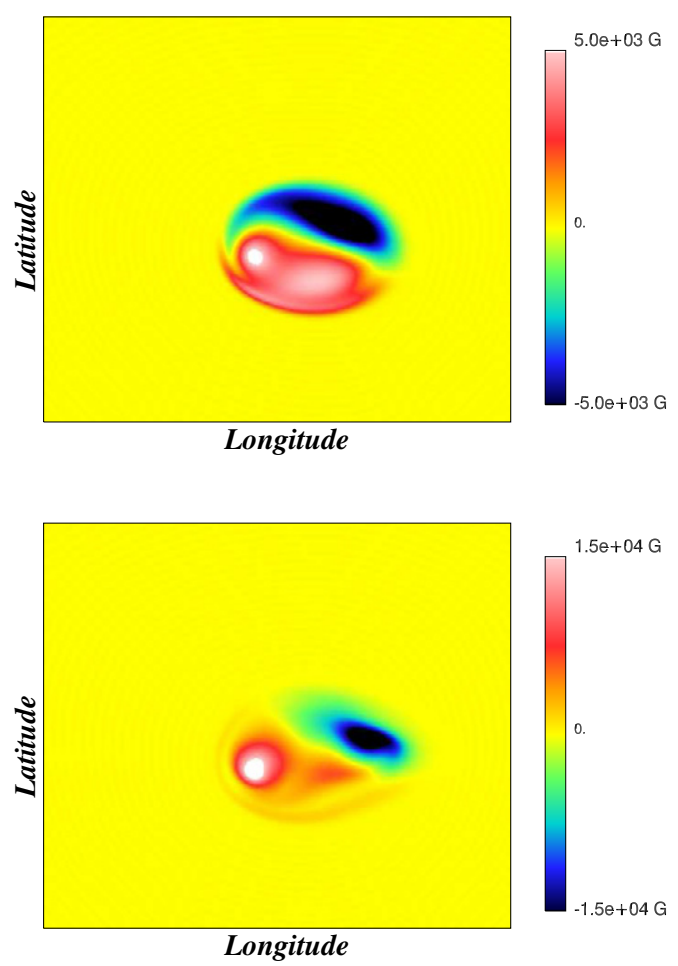

Figure 7. Zoom on the emerging radial field at $r=0.93 R_{\odot}$ for Cases 3TwP and 3TwN where $B_{0}=10^{5} \mathrm{G}$. (A color version of this figure is available in the online journal.)

know from previous studies (e.g., Fan 2008) that the sign of the initial twist will have a dramatic effect on the tilt angle of the emerging region. Here, we address this question by computing Cases 1 and 3 with a right-handed as well as left-handed twist.

Figures 7 and 8 show a zoom on the emerging region in both cases by the time the apex of the loop reaches the top of our domain $\left(0.96 R_{\odot}\right)$. Blue indicates a negative radial field and red a positive. The first thing we note is the typical evolution of the inclination of the bipolar structure. Starting from north/south because of the twist of the loop apex, it becomes more and more east/west as we see the legs of the loop emerging. In agreement with previous thin flux tube calculations (e.g., D'silva \& Choudhuri 1993), we find that a stronger initial field produces a smaller tilt angle since the magnetic tension will tend to oppose the tilting of the magnetic structure. This effect is visible when comparing the left panels of Figures 7 and 8 which differ by a factor two in initial field strength.

The tilt angle of the bipolar structure and the effect of the initial twist is consistent with previous simulations, especially those of Fan (2008). Indeed, in the left-handed case (which is thought to be dominant in the Northern Hemisphere), the tilt angle is not in agreement with observations which tend to show an inclination of the bipolar spots much closer to what we get in the right-handed twist. It was previously argued (Fan 2008) that to reconcile simulations with observations, a very weak left-handed twist had to be introduced so that the Coriolis force acting on the loop would be strong enough to change the tilt angle from a positive to a negative sign. The problem is that such a weak twist, not sufficient to maintain a full coherence of the magnetic structure, leads to a strong flux loss and such structures would be likely to have difficulties rising in a convective environment.
If we look at Figure 8 where the initial field strength is decreased to $5 \times 10^{4} \mathrm{G}$ but the amount of twist kept the same, we manage to get a case where the initial left-handed twist is strong enough to maintain the coherence of the loop and where the tilt angle of the emerging radial field agrees better with observations. The Coriolis force has been able to act sufficiently on this loop to rotate the two opposite polarities clockwise and produce a final tilt angle of the same sign in both cases.

To illustrate this, Figure 9 shows on the same plot the temporal evolution of the tilt angle measured in Cases 1TwP and 1 TwN, at $r=0.93 R_{\odot}$. The tilt angle here was determined by locating the peak values of each polarity and measuring the angle between the line linking them and the East-West direction. At the beginning of emergence, the angle is close to $90^{\circ}$ for both cases, but with opposite signs. As the emergence progresses, the absolute value of the tilt reduces in both cases. At this stage, the effect of the twist is dominant and corresponds to what is measured here. After about 14 days of evolution, the rapid change of tilt stops and the effect of the Coriolis force starts to be visible, especially on Case $1 \mathrm{TwN}$. As a consequence, the curve in Case 1TwP seems to saturate around the value of $55^{\circ}$ and starts to increase again slightly because of the rotation of the spots, to reach a value of about $60^{\circ}$ after 26 days of evolution. In Case $1 \mathrm{TwN}$, the tilt angle continues to increase and crosses the $y=0$ line after about 15 days. At this instant, a change of sign has occurred and the tilt has thus become compatible with observations. A final value of about $15^{\circ}$ is reached after 26 days when diffusion then starts to act, which lies in the acceptable range of values when compared to observations at this latitude.

We now turn to study the influence of latitude on the tilt angle. As we expected, and as seen in Figure 10, the tilt angle depends on the initial latitude since the Coriolis force depends on the 
Positive Twist (right handed)
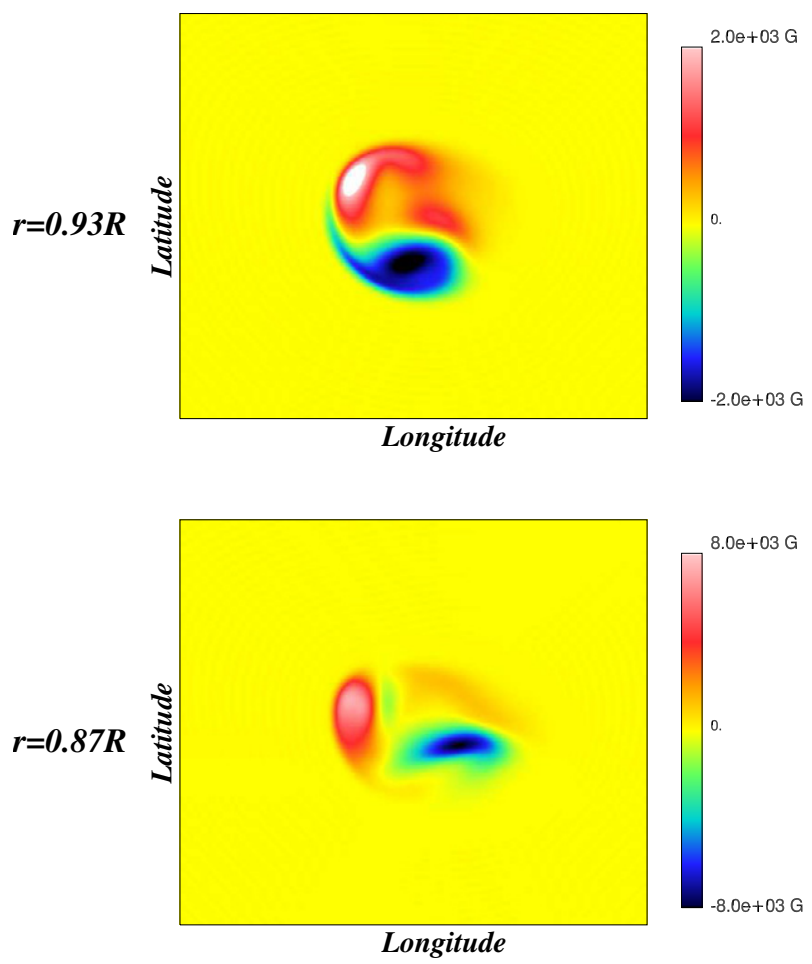

Negative Twist (left handed)
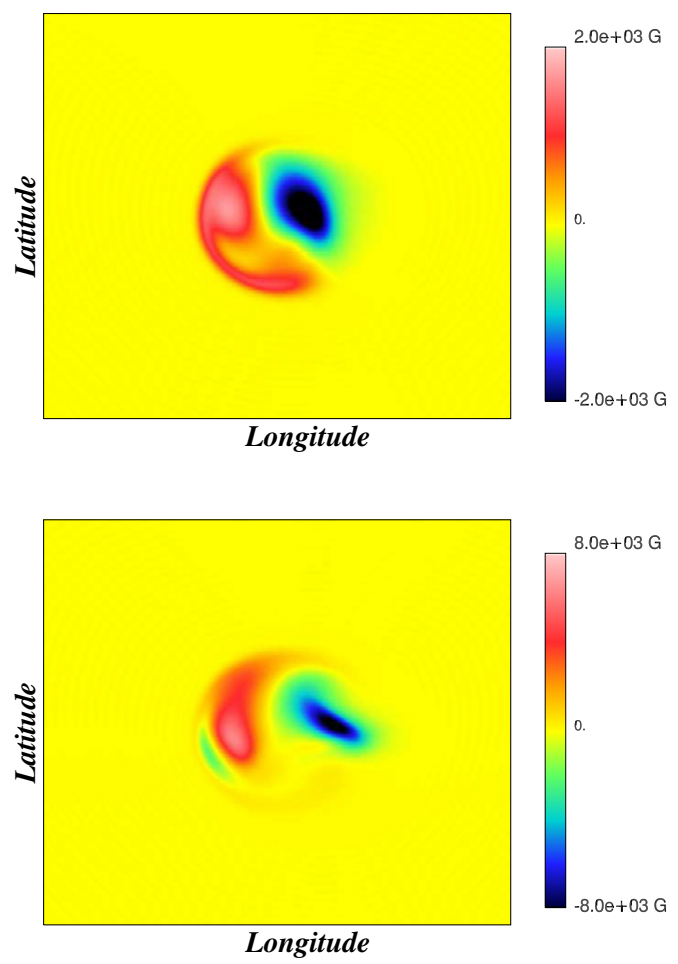

Figure 8. Same as Figure 7, but for Cases 1TwP and $1 \mathrm{TwN}$ where $B_{0}=5 \times 10^{4} \mathrm{G}$.

(A color version of this figure is available in the online journal.)

latitude. From the estimate of D'silva \& Choudhuri (1993), the tilt angle is a decreasing function of the cosine of the colatitude (or an increasing function of latitude), as seen in Figure 10 and as seen in observations. As we saw before in the left-handed case, the bipolar spot will have the tendency to rotate clockwise because of the Coriolis force acting differently on the two legs of the loop. As a consequence, the orientation of the bipolar spots in the case where it was introduced at high latitudes $\left(60^{\circ}\right)$ is close to north/south, and not only because we only see the twist of the field lines here but rather because the axis of the loop has been rotated clockwise by about $75^{\circ}$. When the initial latitude is $15^{\circ}$, the typical tilt angle we get is around $35^{\circ}$, while it increases to $60^{\circ}$ when a loop initially at $30^{\circ}$ is considered. Moreover, we note on this figure that the emergence occurs at slightly different locations in longitude. The center of the bipolar structure is located at around $75^{\circ}$ in Case 1 TwP15 and $90^{\circ}$ in Case 1TwP60. This is due to the asymmetry of the loop which is much more pronounced in the low latitude cases since the action of the Coriolis force on both legs of the loop is stronger then.

We note here that the values for the tilt angle may appear large compared to the classical observations (between $3^{\circ}$ and $10^{\circ}$ typically) and to previous thin flux tube calculations (D'silva \& Choudhuri 1993; Caligari et al. 1995), but as shown before (on Figure 9) an initial left-handed twist, associated with a slow rise allowing the Coriolis force to act on the loop, is able to produce tilt angles of about $15^{\circ}$ in a case where the initial latitude was $30^{\circ}$. This, in turn, is very compatible with observations at the photosphere. It is possible here to make such a comparison if we make the reasonable assumption that the Coriolis force will not have the time to modify the loop orientation and thus the tilt angle during the fast rise through the last few percents of the convection zone.

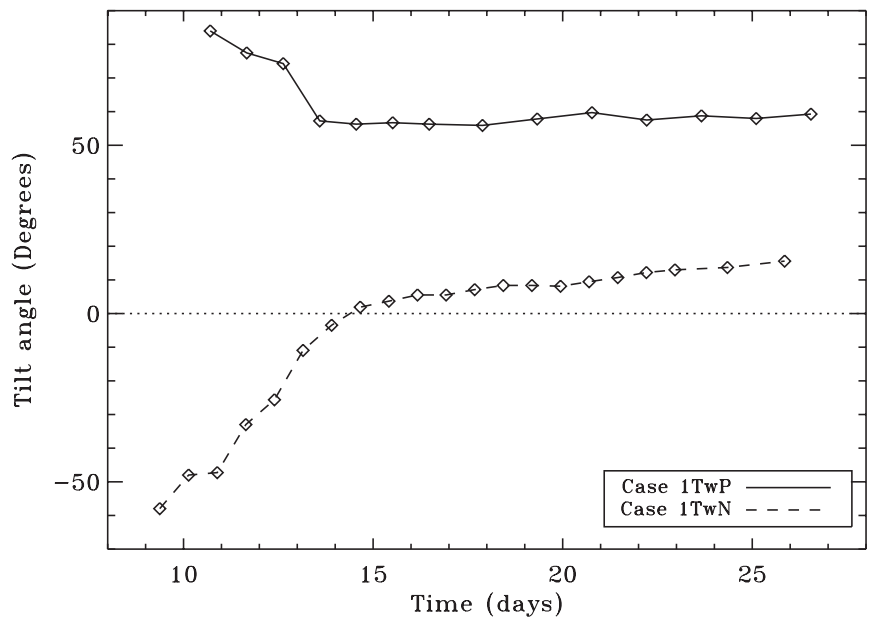

Figure 9. Measure of the tilt angle of emerging bipolar spots for Cases 1TwP and 1TwN, at $r=0.93 R_{\odot}$.

We shall keep in mind those results for the convective cases where the spherical shell will now be differentially rotating. Since the tilt angle is also a function of the local rotation rate, the tilt angles we will get in the convective cases may well be different.

\subsubsection{Morphology of Emerging Regions: Tongues and Necklaces}

If we concentrate again on Figure 7 , then we immediately notice the typical "tongue"-shape of the emerging region, especially at a deeper radius $r=0.87 R_{\odot}$. They are indicated by red and blue arrows in the bottom left panel of Figure 7. Those elongated structures, also seen in the observations (Lopez 


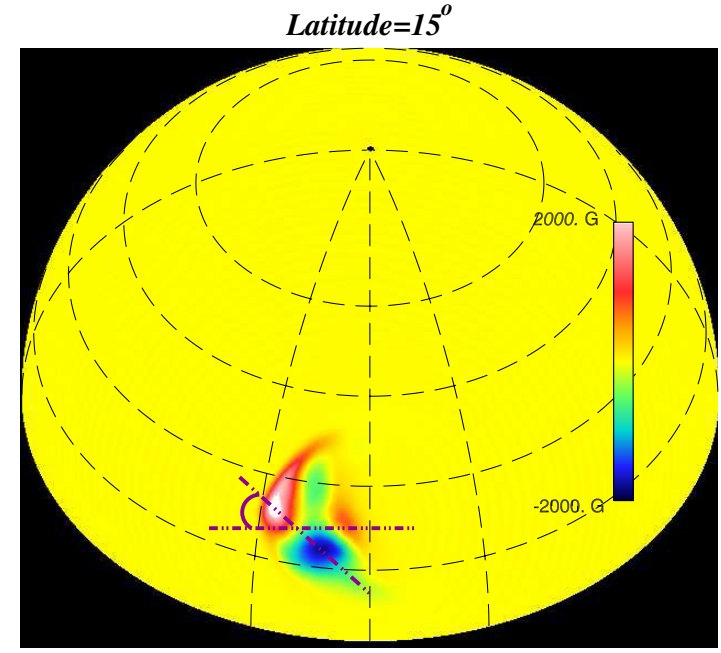

Time $=25.3 \mathrm{~d}$

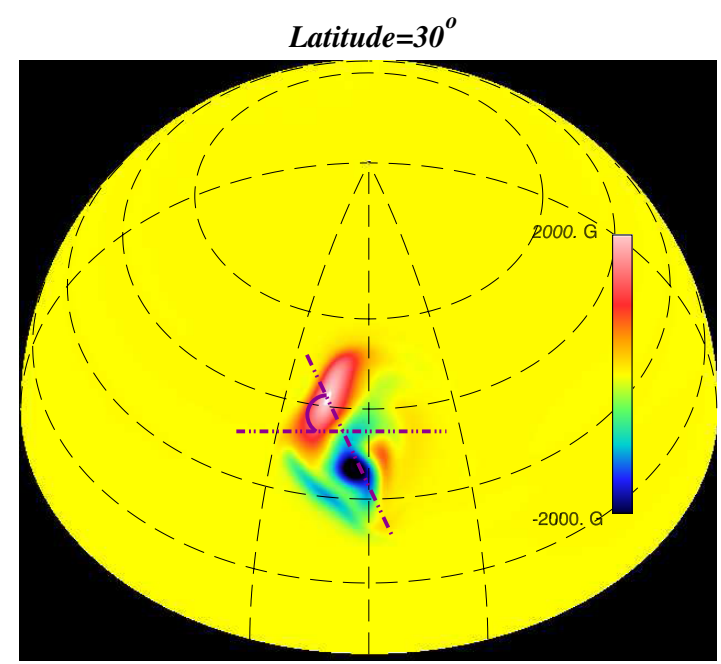

Time $=24.3 \mathrm{~d}$

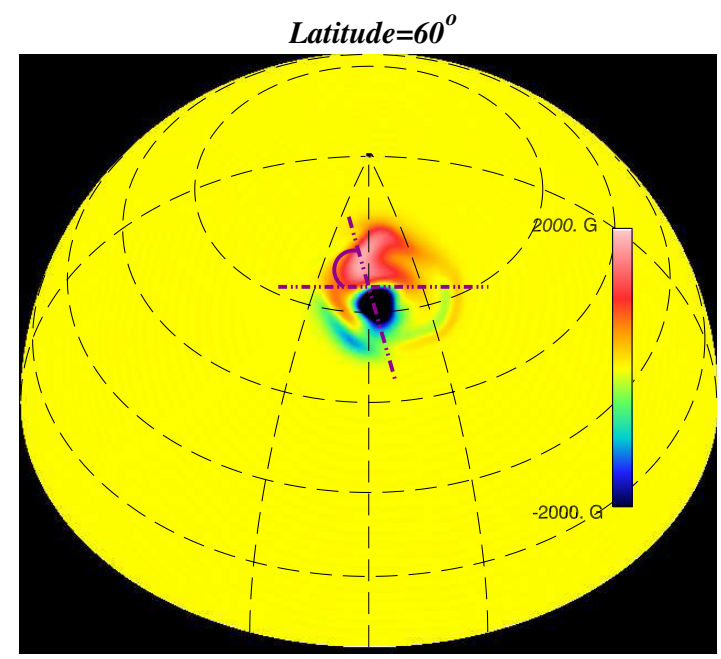

Time $=21.2 \mathrm{~d}$

Figure 10. Measure of the tilt angles of cases where the loop was introduced at the latitudes of $15^{\circ}$ (Case 1TwP15), $30^{\circ}$ (Case 1TwP), and $60^{\circ}$ (Case 1TwP60). The Northern Hemisphere is represented centered at $90^{\circ}$ in longitude and $45^{\circ}$ in latitude.

(A color version of this figure is available in the online journal.)
Fuentes et al. 2000), in Cartesian simulations (Fan 2001; Archontis \& Hood 2010), and studied in detail in Luoni et al. (2011), are mainly due to the twist of the field lines showing in the map of the radial magnetic field. In particular, the orientation of the tongues (or the direction in which they extend) give an indication of the sign of the twist. In the lower left panel of Figure 7, we thus see that both positive and negative polarities extend on their left side, while it is the opposite for the lower right panel where the sign of the twist has changed. Another interesting feature here is the appearance of sharp structures around the emerging region, forming an annular shape that we will from now on call a "magnetic necklace." This annular shape of emerging active regions is also often seen in observations but has never been reported as identified magnetic structures, different from the so-called tongues. This is true for observations with experiments and instruments as varied as the Flare Genesis Experiment (Pariat et al. 2004), Hinode (Otsuji et al. 2011), or SOHO/MDI (Liu \& Zhang 2006).

Those structures are clearly visible in the lower panels of Figure 7 but do not really show in Figure 8 because of the low field strength and the rather large magnetic diffusivity in our simulation. Indeed, since those structures are organized at rather small scales, it is necessary to have low enough diffusivities to be able to see those structures at the time of emergence and during their rise. As a consequence, we decided to compute Case 1TwN with a lower magnetic diffusivity (we choose $P m=5$ here) to see if these small-scale structures could be recovered in this case. Indeed, as shown in Figure 11, the magnetic necklace is visible on the zoom in the $(\theta, \phi)$ plane on the emerging region at $0.93 R_{\odot}$ and is also seen in a meridian cut of the loop during its rise (see red and blue arrows in the left panels). They consist of sharp elongated structures (not to be confused with the tongues) lying at the periphery of each polarity and of the same sign that create a ring inside which the two main regions of strong radial field concentrate. If we compare the regions of appearance of these sharp structures around the bipolar spot with the norm of the vorticity (or enstrophy), it is rather clear that those structures correspond to a velocity shear which produces strong vorticity. As shown and studied in Emonet \& Moreno-Insertis (1998), a sharp interface is indeed formed at the boundaries of the flux rope while it rises. This region is the site of vorticity generation via the presence of a magnetic field during the whole loop evolution. Another source of vorticity exists within the tube interior and is linked to the gravitational torque applied between the tube center and its periphery. This process creates the counter-rotating vortices which can completely break up the tube during its rise if the twist (and thus the magnetic tension) is not strong enough, as seen in Section 4.1.1. Looking at the four panels of Figure 11, we understand that the necklace, associated with vorticity generation at the loop edges, will be visible only after a significant part of the loop has emerged. Indeed, since the main vorticity generation is located at the sides of the loop, we need to wait until the axis of the loop crosses the surface $r=0.93 R_{\odot}$ before being able to observe the sharp magnetic structures. By that time, the tongues have already significantly faded away and reconcentrated around the two polarities. We can thus expect to see those structures in actual observations only after a certain amount of time following the first signs of emergence.

\subsubsection{Field Strengths and Fluxes}

We end this section by noting another difference between Cases 1 and 3. In Case 3, the loop seems to maintain more 
Radial Magnetic field
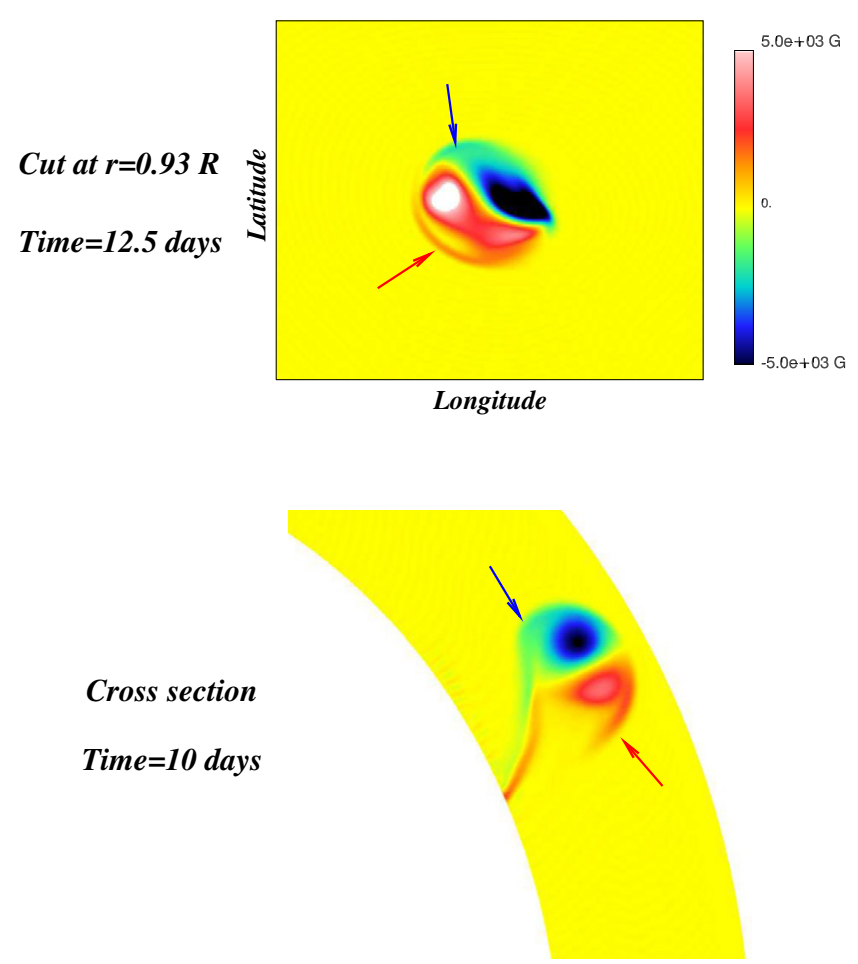

Norm of Vorticity
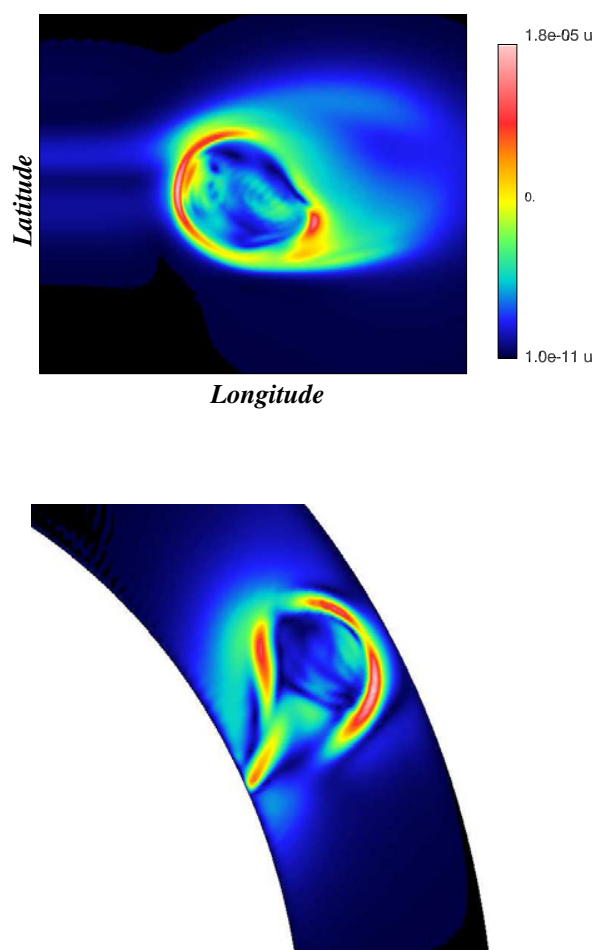

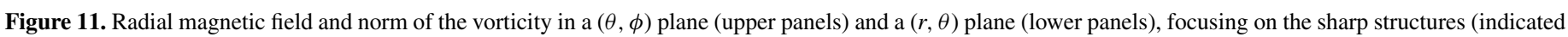
by arrows) that surround the emerging regions.

(A color version of this figure is available in the online journal.)

coherence while it rises, both polarities are well-separated, even at the end of the simulation when the bipolar region reaches its maximum extension. This is less the case for the weaker field of Case 1. Indeed, both polarities seem to get mixed and the radial magnetic field lines are more easily advected by the velocity field created by the loop itself (we remind that there is no convection in this case). At the end of the simulation, the magnetic field is advected at the boundary of the positive radial velocity pattern it creates and finally diffuses away.

If we now focus on the more realistic Case 1 (with a field strength of $5 \times 10^{4} \mathrm{G}$, and a typical rise time of more than 10 days), we can move to a more quantitative study of the evolution of the intensity of the radial field emerging in each spot and of the amount of flux it represents. The results of this study are shown in Figure 12. Panels (a) and (b) show the evolution, during emergence at $r=0.93 R_{\odot}$ of the radial field and radial flux in Case 1TwP, and panels (c) and (d) in Case 1TwN. In both cases, at the beginning of emergence, the negative field in the leading polarity dominates over the trailing polarity (by about $35 \%$ ), while the amount of flux is the same in both polarities (since all emerging field lines of one polarity connect to the other one in the domain of integration). This suggests that the leading polarity is stronger in intensity and more concentrated and the trailing polarity is weaker and less coherent, which is in agreement with observations of emerging active regions (e.g., Kosovichev \& Stenflo 2008). Moreover, this feature was also first seen in Cartesian simulations and explained in Fan et al. (1993) by the evacuation of plasma out of the leading side of the loop into the following side. The leading side then shrinks and the magnetic pressure increases to balance the same external pressure, resulting in a more intense field in the less extended leading polarity. As Fan et al. (1993) point out and as is discussed in Caligari et al. (1998), the anchoring of the field lines outside the buoyant part of the loop is a key ingredient in producing a strong asymmetry in field strength. It is also what we find here in Case $1 \mathrm{TwN}$, for example, where a strong asymmetry in inclination develops because of the anchoring of the field lines outside the most buoyant part and where we find a leading leg about 1.4 times more intense than the trailing leg, on average (see panel (c) of Figure 12).

We note that the values of the flux are quite strong compared to observations (which report values of about $10^{21}-10^{22} \mathrm{Mx}$ ). This is due to the scales of our magnetic structures which overestimate the size of a flux tube initially located at the base of the convection zone. The initial flux in the magnetic structure in Case 1 is $6 \times 10^{23} \mathrm{Mx}$. Simulations with flux tubes with radii that are about 10 times smaller, numerically very challenging, would lead to values for the fluxes that are much closer to observations (Schrijver \& Harvey 1994). It is however important to note that despite the discrepancies between the absolute values of the fluxes, due to numerical limitations, the shape of the temporal evolution of the fluxes is in good agreement with observations. Indeed, the flux in each polarity of the bipolar active regions is observed, on Michelson Doppler Imager (MDI) magnetograms for example (van Driel-Gesztelyi et al. 2003), to increase sharply in the first days of emergence, before saturating and slowly starting its diffusive decay. We get exactly the same kind of evolution in our simulations, visible especially in panel (b) of Figure 12 . The flux strongly increases from 0 to $1.5 \times 10^{23} \mathrm{Mx}$ in the first five days before saturating around this value. In the figure and especially in panel (d), we distinguish first a slow rising phase between 8 and 9 days, followed by the very sharp 

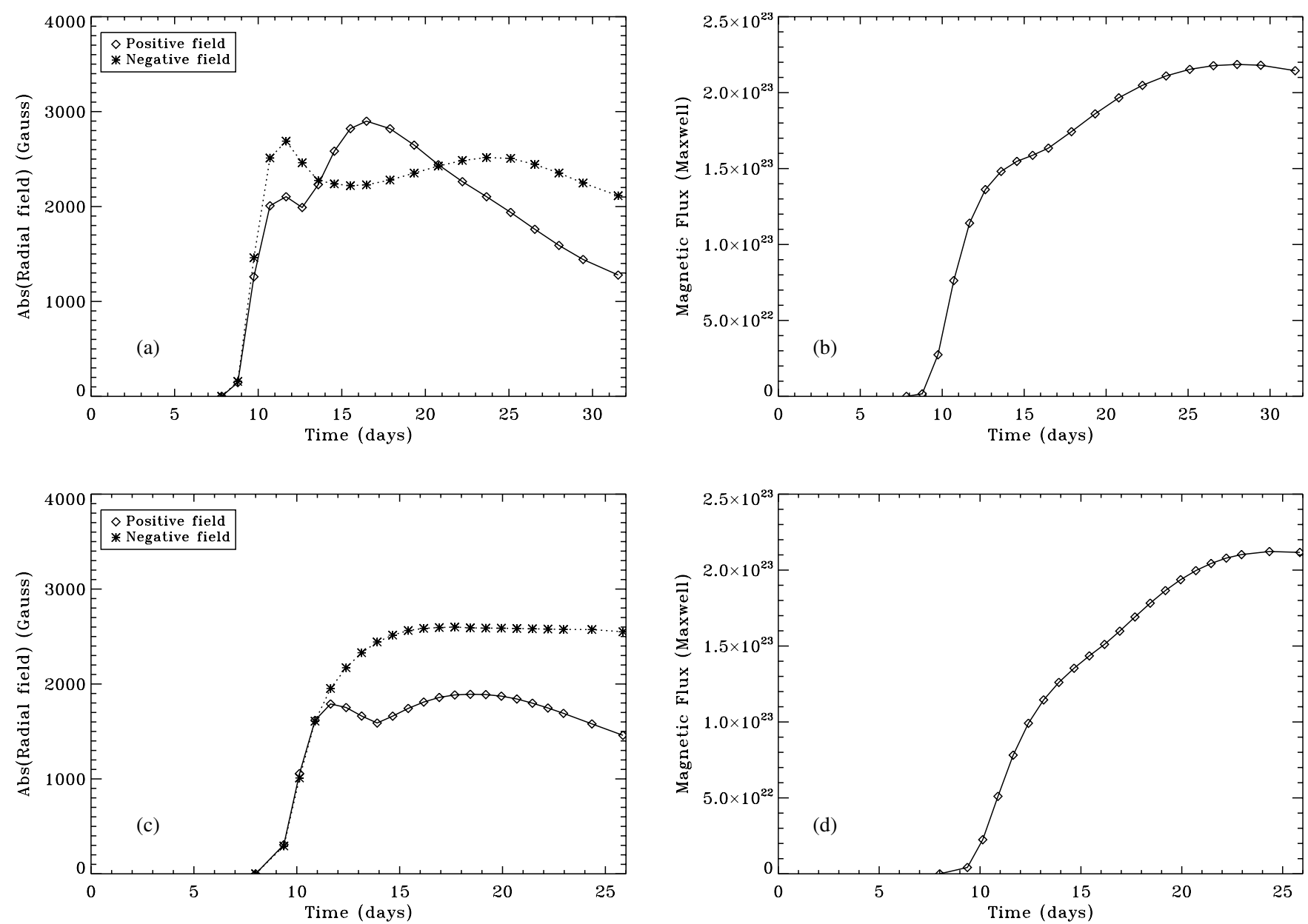

Figure 12. Radial field and unsigned radial flux for Case 1 with right-handed (panels (a) and (b)) and left-handed (panels (c) and (d)) initial twist. The values of the field are measured at $r=0.93 R_{\odot}$ in the center of each polarity where the field is strongest and the fluxes are the integrals of the radial field contained in the trailing (or leading) polarity through a surface enclosing the emerging region.

increase of the amplitude of the flux after 10 days of evolution. The first phase is related to the very beginning of the emergence when only the apex of the loop is visible and the only contributor to the flux is the radial magnetic field coming from the twist of the field lines. As the emergence proceeds, however, the feet of the loops where the strong radial magnetic field lies start to emerge and form the bipolar region. It is thus this fast emergence phase of the arched structure that produces the sharp increase of the simulated (and possibly of the observed) magnetic fluxes. As far as the magnetic field strength is concerned, we can wonder what typical horizontal field strength we would get at the photosphere, starting with our flux tubes located at the base of the CZ, in the same way as Cheung et al. (2010). If we follow the evolution of the axial field intensity in time (and thus in radius), we find that the scaling with the reference density is close to $B \propto(\bar{\rho}+\rho)$ (similar to what is found in Pinto \& Brun 2012), corresponding to an expansion of the loop in the directions transverse to its axis. At the top of our domain, the typical toroidal (or horizontal) field is of the order of $10 \mathrm{kG}$ (for Case 1TwP for example) and the density $\bar{\rho}$ of the order of $4 \times 10^{-3} \mathrm{~g} \mathrm{~cm}^{-3}$. As a consequence, if we consider a density of $4 \times 10^{-7} \mathrm{~g} \mathrm{~cm}^{-3}$ at the photosphere, then the horizontal field strength would reduce to $1 \mathrm{G}$, much weaker than the typical observed values (around $100 \mathrm{G}$ ). However, the expansion in the last few percent of the convection zone might be modified (becoming mostly horizontal, for example, due to a decrease of the plasma $\beta$ ), and then the scaling with density could change drastically and become $B \propto \sqrt{\bar{\rho}}$, as shown in Cheung et al. (2010). We should then keep in mind that we are not modeling the uppermost layers of the convection zone and that it is not relevant here to relate directly the field strengths in our bipolar regions to the field strengths which would be reached at the photosphere.

Comparing the left-handed and right-handed twist cases, we find that the evolutions, especially for the radial field, are slightly different. Indeed, the dominant leading polarity at the beginning of emergence in Case 1TwP (panel (a)) saturates at the value of $2700 \mathrm{G}$ and then drops to the value of $2300 \mathrm{G}$ while the trailing polarity continues to increase in intensity. At $t=14$ days, the positive trailing polarity thus becomes dominant, before decreasing at $t=21$ days. This is not the case for Case 1TwN (panel (c)), where the leading polarity always stays dominant in strength and approximately equal to the trailing polarity in flux. This could be due to the asymmetry built up during the rise, which is smaller in the left-handed twist case. This is indeed seen on cuts at constant latitude, as in Figure 5 (not shown here). As a consequence, in Case 1TwP, the asymmetry between the legs is strong, the leading polarity reaches its maximum strength, and thus starts to diffuse away before the trailing polarity. This explains the decrease in amplitude in Figure 12 first seen for the negative field and then for the positive field. In Case $1 \mathrm{TwN}$, the peak positive and negative radial fields are 


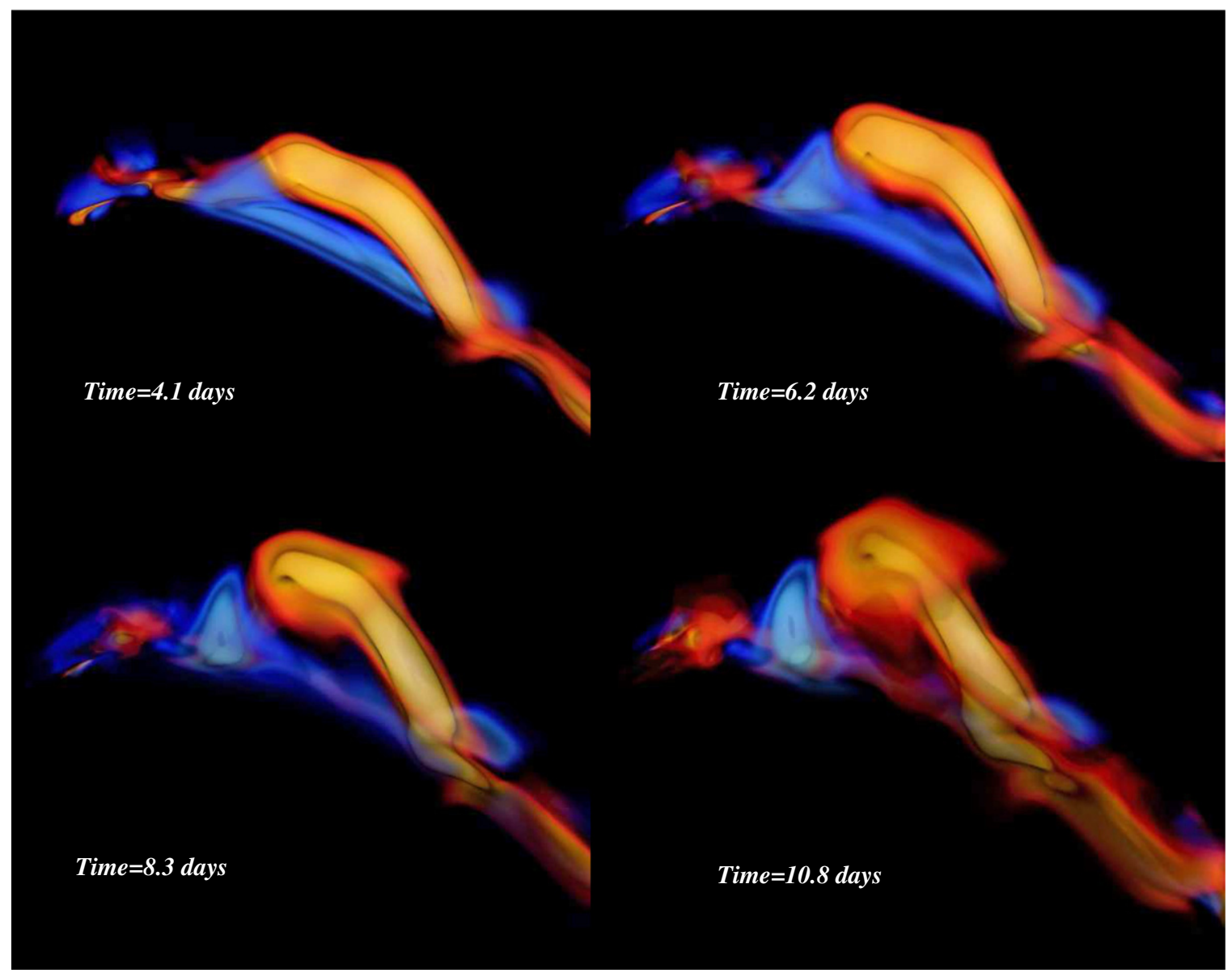

Figure 13. Volume rendering of $B_{\phi}$ while the loop rises through a convective layer. As in Figure 4, the loop is viewed from below, looking up at the North Pole. The deformation of the loop by convective motions is clearly visible here when compared to Figure 4.

(A color version of this figure is available in the online journal.)

measured at $r=0.93 R_{\odot}$ at approximately the same time since the asymmetry is less pronounced and they both saturate at the same time (at the value of $1900 \mathrm{G}$ for the positive polarity and $2500 \mathrm{G}$ for the negative one), before starting their diffusion.

\section{EVOLUTION OF A SINGLE LOOP WITH CONVECTION}

We now turn to investigate the behavior of a loop similar to the cases presented above, but inside a convective background. As in the global flux tubes simulations (Jouve \& Brun 2009), the buoyancy force will compete with the downflows to drive the loop toward the top of the computational domain and the emerging region will have significantly different characteristics from the isentropic cases. Figure 13 shows the typical threedimensional evolution of an $\Omega$-loop evolving in a convective environment, which corresponds to Case 2TwP. We only see here the evolution of the toroidal field but a turbulent convective velocity field exists all around it. Moreover, differential rotation and meridional circulation have well-established profiles here, as stated in Section 2.3.

We recover some aspects of the cases studied in Section 4, like the asymmetry between the legs and the creation of a negative toroidal field at various regions at the loop periphery. Again, a local $\Omega$-effect is at work here, with a slightly more complex structure since the velocity field is now organized at different spatial and temporal scales. But the main factors (azimuthal velocity gradients and radial and latitudinal components of the magnetic field) are still the same and able to locally create negative toroidal field.

\subsection{In the Bulk of the Convection Zone}

In such a fully convective setting, we expect the initial field strength in the flux tube to be an important parameter for the evolution and rise of the magnetic field. Indeed, as shown in previous work involving a uniformly buoyant flux tube (Fan et al. 2003; Jouve \& Brun 2009), the downflows and upflows of the convective layer control the rise velocity of the tube and are sometimes even able to pin it within the convection zone, resulting in structures emerging at specific longitudes. Here again, we get the same kind of competition between convection and magnetic buoyancy, which can be seen in Figure 14. This figure shows the evolution of the loops of Case 1TwP and Case 2TwP. They correspond to the second and third panels of Figure 5, for which the asymmetry was clearly modified when the parameters were changed so as to have a more intense field. In Case 1 , the initial field strength is $5 \times 10^{4} \mathrm{G}$, i.e., slightly below the equipartition field strength of $6 \times 10^{4} \mathrm{G}$, corresponding to the magnetic field in equipartition with the strongest downdrafts at the base of the CZ. In Case 2, the initial magnetic field strength is $10^{5} \mathrm{G}$, corresponding to 1.7 times the equipartition field. Here, we superimpose the magnetic energy contours onto the background radial velocity (representing the convective motions). For Figure 5, we chose to represent the loops at about $t=10$ days, since their position in the bulk of 
Case 1TwP
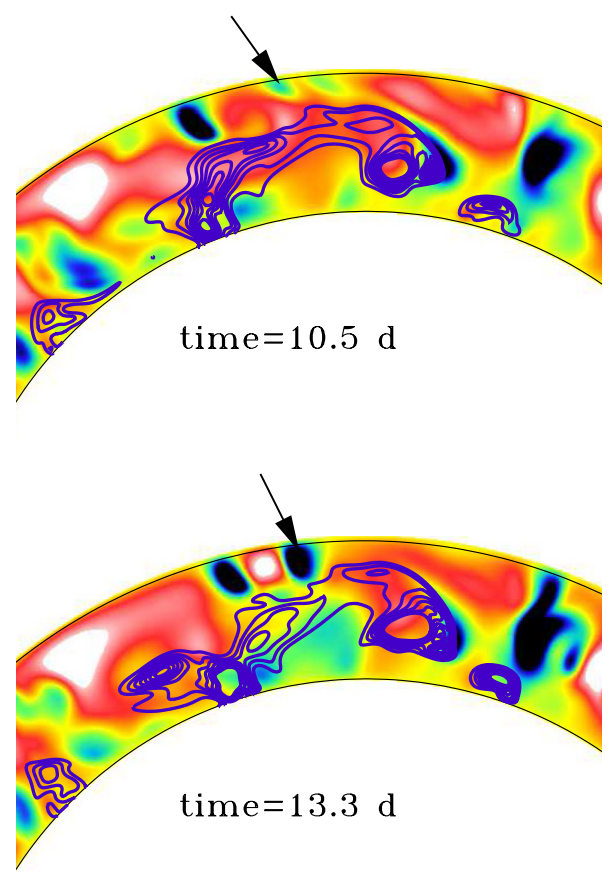

Case2TwP

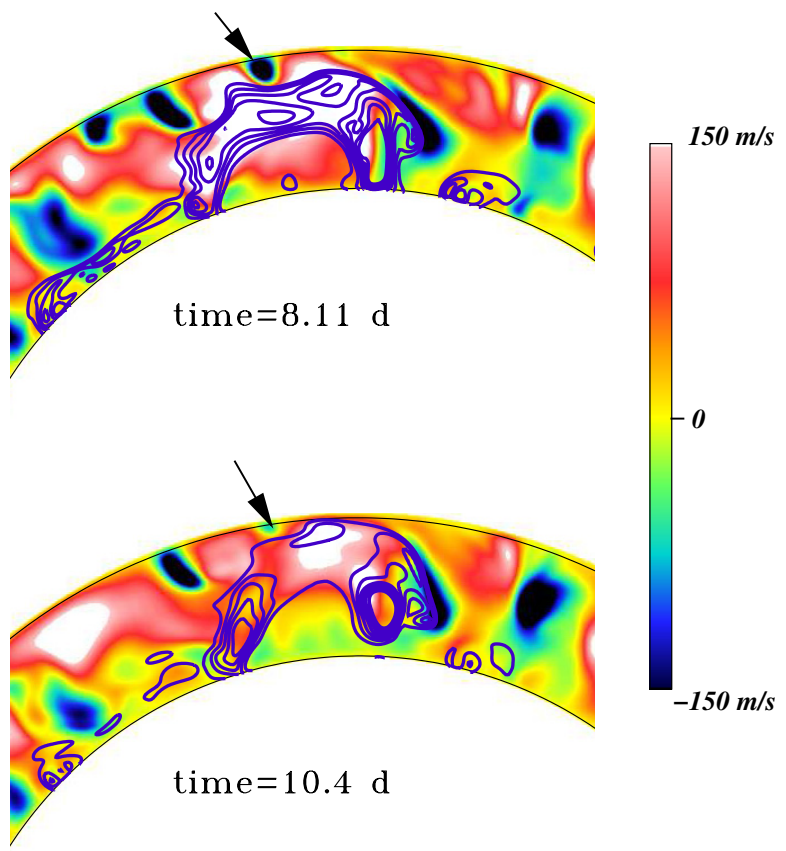

Figure 14. Cut at the latitude of $30^{\circ}$ of the magnetic energy (contours) superimposed onto the background radial velocity (blue meaning downflows and red meaning upflows) for Cases $1 \mathrm{TwP}$ and $2 \mathrm{TwP}$. The color bar indicates the values taken by the radial velocity.

(A color version of this figure is available in the online journal.)

the $\mathrm{CZ}$ was not very different for the isentropic cases. Here, at about $t=10$ days, the loops have not reached the same height, the apex of the loop of Case $2 \mathrm{TwP}$ has reached $r=0.93 R_{\odot}$ after about 8 days already, whereas the loop of Case 1TwP reaches the same height at $t=13$ days and was thus significantly slowed down compared to the isentropic case. This can be explained by the structure of the flow in which the loops are embedded. In Case 2TwP and as seen especially on the panel at time $t=8.11$ days, the velocity field associated with the Lorentz force is dominant. At the location of maximum magnetic field, a strong upflow thus develops (a maximum positive radial velocity of $350 \mathrm{~m} \mathrm{~s}^{-1}$ is reached here), the loop is dragged upward and the influence of the downflow shown by the black arrow is limited. The rise of the loop is thus widely promoted by the strong upward convective cell produced by the intense magnetic field. On the contrary, in Case 1TwP, where the magnetic energy content (and thus the buoyancy) is weaker, the radial velocity created by the presence of a magnetic field is of the same order as the background convective motions and the downflows (as the one indicated by the arrow) are much more efficient at maintaining the magnetic loop under the surface and preventing the axis from emerging.

Another difference we note here compared to the isentropic cases is the degree of asymmetry between the trailing and leading legs. The strong asymmetry visible in Case 1TwP when the loop was embedded in an isentropic background is still present at $t=13.3$ days, but it is less obvious since the advection by the convective motions has also mixed the field lines and significantly modified the structure. However, in Case 2TwP at $t=10.4$ days, the same degree of asymmetry as in the isentropic case is found, showing again that this regime is dominated by the Lorentz force, contrary to Case 1TwP where the magnetic

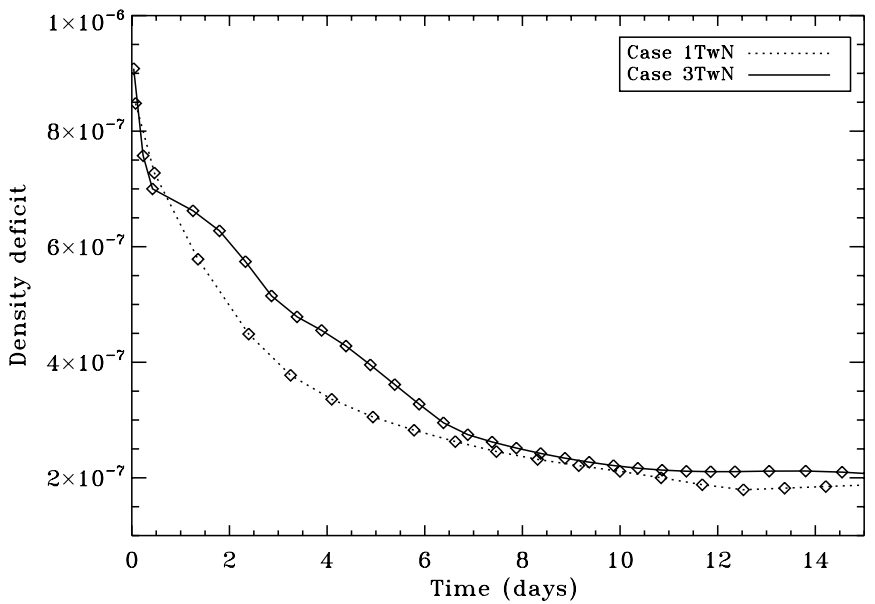

Figure 15. Density deficit at the apex of the loop during its rise in Cases 1TwN (dotted line) and 3TwN (solid line). The internal density is measured inside the tube apex, where the magnetic field is maximum and compared to the external density which is taken to be the average value of the density at the corresponding depth.

structure is essentially frozen in (advected by) the convective motions.

In order to gain a better insight as to why we get such different behavior between what we will now call the convectiondominated regime of Case 1TwP and the magnetic field dominated regime of the other cases, we plot the evolution of the density deficit inside the loop at its apex as a function of time in Cases 1 and 3. The results are shown in Figure 15. They correspond to Cases $1 \mathrm{TwN}$ and $3 \mathrm{TwN}$ but the results are similar for the right-handed twist cases. Our results here, and especially 
the distinction between convection and magnetic field dominated cases, are similar to what is obtained in the recent thin flux tube simulations of Weber et al. (2011). Indeed, they find that an initial field strength above $10^{5} \mathrm{G}$ ensures an evolution dominated by magnetic buoyancy while a loop initialized with $B_{0}=1.5 \times 10^{4} \mathrm{G}$ is strongly influenced by convective motions. Similar values are found here.

Figure 15 shows the evolution of the density deficit inside the loop compared to its surroundings for Case 1 (dotted line) and Case 3 (plain line). This density deficit represents the buoyancy of the structure and thus its capacity to reach the top of our computational domain. Both loops started with approximately the same density deficit, as seen at the beginning of the evolution. The two cases initially follow the same amount of buoyancy loss during about half a day and then the two cases start to have different behaviors. Indeed, in Case 1, the loop continues to smoothly lose its density deficit while the simulation proceeds and follows the exponential decay of a typical solution of diffusion equation. After $t=10$ days, the density fluctuations in the bulk of the convection zone are of the same order as the density deficit in the loop and the magnetic structure stops being buoyant. We also note that convective motions act on the magnetic structure to create smaller scale regions that will thus diffuse faster and lead to a smaller field strength and thus smaller buoyancy. At $t=15$ days, the loop still has not reached the top of the domain and the only reason why we will get some emerging magnetic field in this case is that upflows will drag the loop upward. The coherent bipolar structure will then be mostly lost when the magnetic structures emerge at $r=0.93 R_{\odot}$, as we will see in the following section. In Case 3, on the contrary, the evolution of the density deficit is quite different. The major trend is of course a decrease of the buoyancy of the structure, but this decrease does not follow a decaying exponential as it had in the previous case. Here, strong currents generated by the higher field strength (initially $10^{5} \mathrm{G}$ ) will play a role in the energy balance of the loop and the diffusion of entropy will be less efficient in this case. As we see in the figure, the buoyancy of the loop of Case 3 always stay higher than in Case 1. As a consequence, at $t=15$ days, a significant part of the loop has emerged at the surface as a coherent bipolar structure. The loop thus keeps both its coherence and its buoyancy for a longer time and the structure of the emerging regions will tend to be less influenced by the convective motions.

\subsection{Structure of the Emerging Regions}

As stated in the introduction, one of the main questions we wish to answer in this work is the following: "what is the influence of convective motions and large-scale flows on the structure of our emerging magnetic field?" Although our top boundary condition is still $28 \mathrm{Mm}$ below the surface and the density and pressure drops may well cause large modifications to the observed magnetic field, we can get some insight into what type of active regions may be created at the solar surface from our numerical simulations.

\subsubsection{Convection versus Magnetic Field Dominated Regimes}

In this section, we focus on the convection-dominated regime of Case 1TwP (and 1TwN), where the emerging magnetic field will be strongly affected by the convective motions. Figure 16 shows four snapshots of the emerging region zoomed in latitude and longitude, at $r=0.93 R_{\odot}$. The radial velocity (colors) represents the convective motions (blue downdrafts and yellow upflows) and we superimpose the emerging radial field (red contours indicating positive polarity). At the beginning of emergence at about $t=9$ days, a well-defined bipolar structure can be identified, at a latitude of $30^{\circ}$, indicating that the rise was indeed radial and not parallel to the rotation axis. We note already on this snapshot that the strong downflow located at the latitude of $30^{\circ}$ and the longitude of $105^{\circ}$ strongly interacts with the negative polarity and is responsible for its cashewnut-like shape. As the emergence proceeds, the well-identified bipolar structure is replaced by a much more complex field structure. The negative polarity gets squeezed into the previous strong downflow, while regions of positive polarity start to emerge all around. The concentration of the magnetic field in the downflow lanes is even more visible in the third snapshot at $t=16.1$ days, where the field concentration seen on the previous panel continues to accumulate in the downdraft while a new emerging bipolar structure appears at the longitude of $90^{\circ}$ where a large upflow was already present and responsible for this new emergence. We then note that this new region is quickly advected toward the boundary of this large upflow and, in turn, is concentrated into the neighboring downdrafts, leading to an evolution very close to what is observed in simulations of magnetoconvection (Weiss et al. 1996). In this case, convective motions were thus very weakly affected by the emerging magnetic field and completely dominated its evolution.

The evolution shown in the previous figure is quite different when cases with stronger buoyancy and a stronger initial field strength are considered. We now turn to investigate the structure of the magnetic field emerging in Cases 2TwP and 4TwP, which initially possess the same buoyancy but different field strengths $\left(10^{5} \mathrm{G}\right.$ for Case 2TwP and $1.35 \times 10^{5} \mathrm{G}$ for Case 4TwP). These values, respectively, correspond to 1.7 and 2.3 times the equipartition field strength. The results are shown in Figure 17. Four snapshots are shown, two for each case, at approximately the same times, $t=9$ days and $t=11$ days. Case 2TwP is interesting since it is an intermediate case between convection-dominated and magnetic field dominated evolution. Indeed, at the beginning of emergence, a bipolar structure emerges at about $95^{\circ}$, as expected (and as found in the isentropic cases), but another region, which is in fact another part of the loop, also emerges at a longitude of $105^{\circ}$. This is due to the strong downflow lane at about $100^{\circ}$ of longitude and its neighboring upflow which will have the effect of dragging the loop upward in the upflow and pinning it down in the downflow (those flows are also visible in Figure 14). As a consequence, two separate regions seem to emerge when they are part of the same $\Omega$-loop but strongly influenced by convection. As the simulation evolves and emergence proceeds, we observe that the magnetic field is advected in the downflow lanes at the largest longitudes but takes shape as a typical active region at around $90^{\circ}$. Indeed, the tongue-shape appears here, although modified by the convective motions. This typical case, intermediate between a convection-dominated and magnetic field dominated evolution, is also interesting since it directly relates to observations of emerging regions. Indeed, the regions of strong magnetic field in the center of the active regions are only marginally affected by the convective motions, as opposed to the surrounding plage regions whose structure is affected by the supergranular scales of convection. We could thus consider that the parameters of Case 2 enable us to simulate an emerging region with characteristics typical of an observed active region, in particular its field strength in each polarity, its competition with convective motions, and its tongues or tilt angle. 
Case 1TwP: Convection-dominated
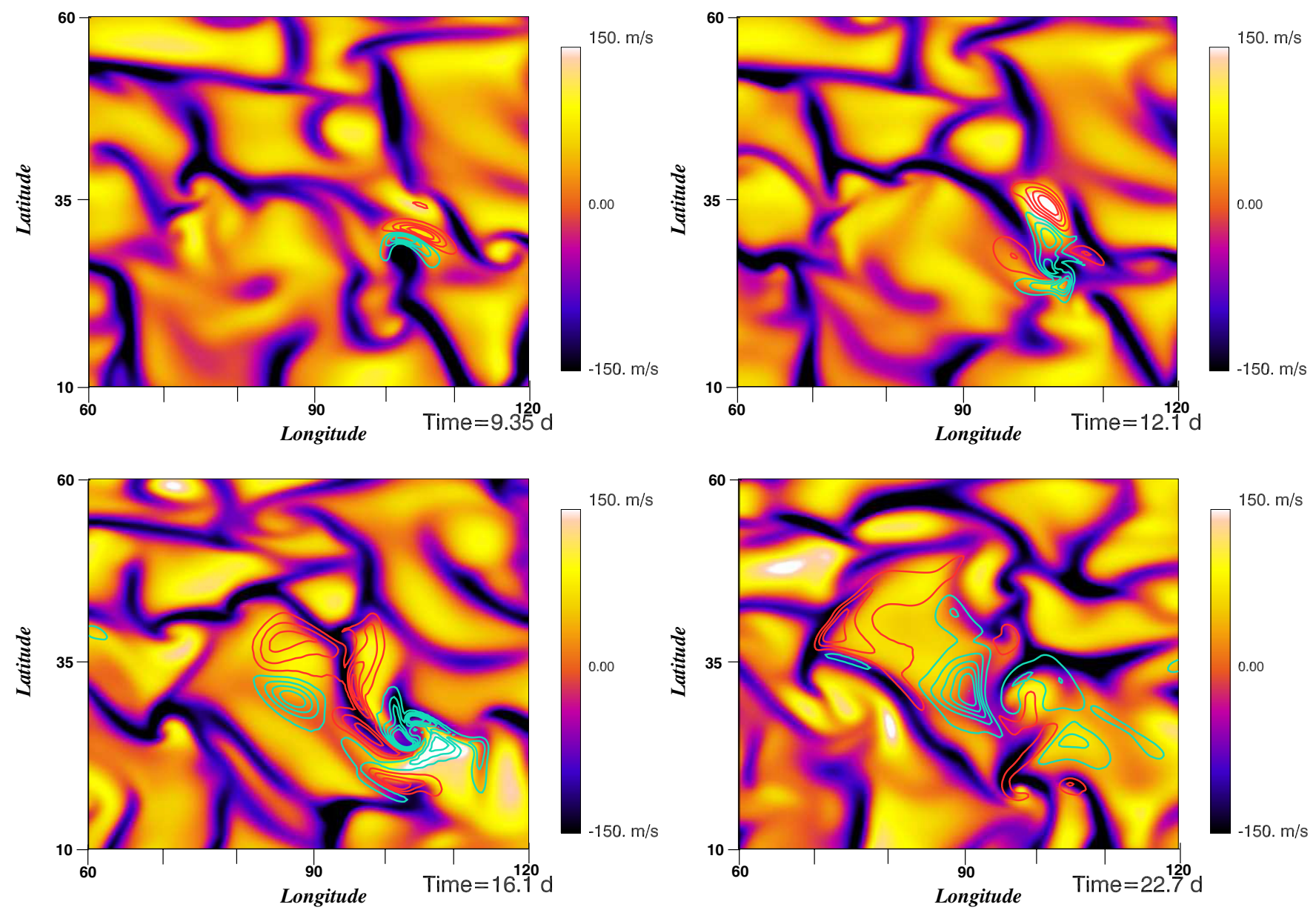

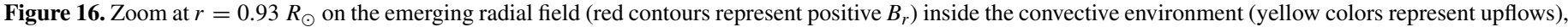
in Case 1TwP. The typical magnetic field strength in these snapshots is about $2000 \mathrm{G}$.

(A color version of this figure is available in the online journal.)

If we now look at Case 4TwP, where the initial magnetic field was stronger, the emergence is much more similar to what we had in the isentropic cases. The orientation of the unique (unlike Case 2) structure shifts from first North-South to more and more East-West, the tongues are easily identified, and a strong upflow is created by the loop itself and dominates its evolution during the emergence process. We observe in Figure 17 that the apex of the loop emerges at a longitude of $95^{\circ}$ and that no radial field is visible at $105^{\circ}$ in Case 4, contrary to Case 2. This is due to the fact that in Case 2, the advection of the magnetic structure by the convective motions significantly modifies the shape of the loop and thus the asymmetry between the trailing and leading legs. In Case 4, the evolution is again closer to the isentropic case and convection has little influence on the loop and is thus not strong enough to produce an emerging region within the upflow located at $105^{\circ}$. In conclusion, although Case 2 is more influenced by convection, a clear impact of the emerging structure on the velocity field is visible in both cases, especially at $t=11$ days where a strong upflow is created at the very location of emergence.

\subsubsection{Effect of Twist and Latitude}

In this last section, we investigate the effect of changing the sign of the twist of the initial magnetic loop, as well as modifying its latitude of introduction. We saw in the isentropic cases that the sign of the twist would influence the tilt angle of the emerging region but that a tilt compatible with observations could still be found with an initial left-handed twist. We now address the question of the possibility of getting tilt angles that are even more compatible with observations when the magnetic loop is embedded in a convective environment.

Before specifically studying the tilt induced by the twisted structures, we investigate the impact of a negative initial twist on the intensity of the emerging radial field. Indeed, it was already found in the isentropic cases that the strength of the emerging polarities and the positive and negative fluxes had a different evolution when a left-handed or right-handed twist was considered. This is still the case when the loops are introduced in a convective shell. Figure 18 presents the evolution of the radial field of the leading and trailing polarities at the time of and after emergence for Cases 1TwP, 1TwN, 3TwP, and 3TwN. Similar pictures for Cases 1TwP and 1TwN were shown in Figure 12 for the isentropic simulations. We can thus first compare the convective and isentropic situations for Case 1. The first thing we note, and which is true both for the right-handed and lefthanded twist, is that the loop in the isentropic case had a quicker rise than in the convective case. Similar results were found for uniformly buoyant flux tubes in Jouve \& Brun (2009). Indeed, for example, at $t=10$ days for the isentropic cases, the typical field strength already reached $2000 \mathrm{G}$, whereas it barely gets to 
Case 2TwP
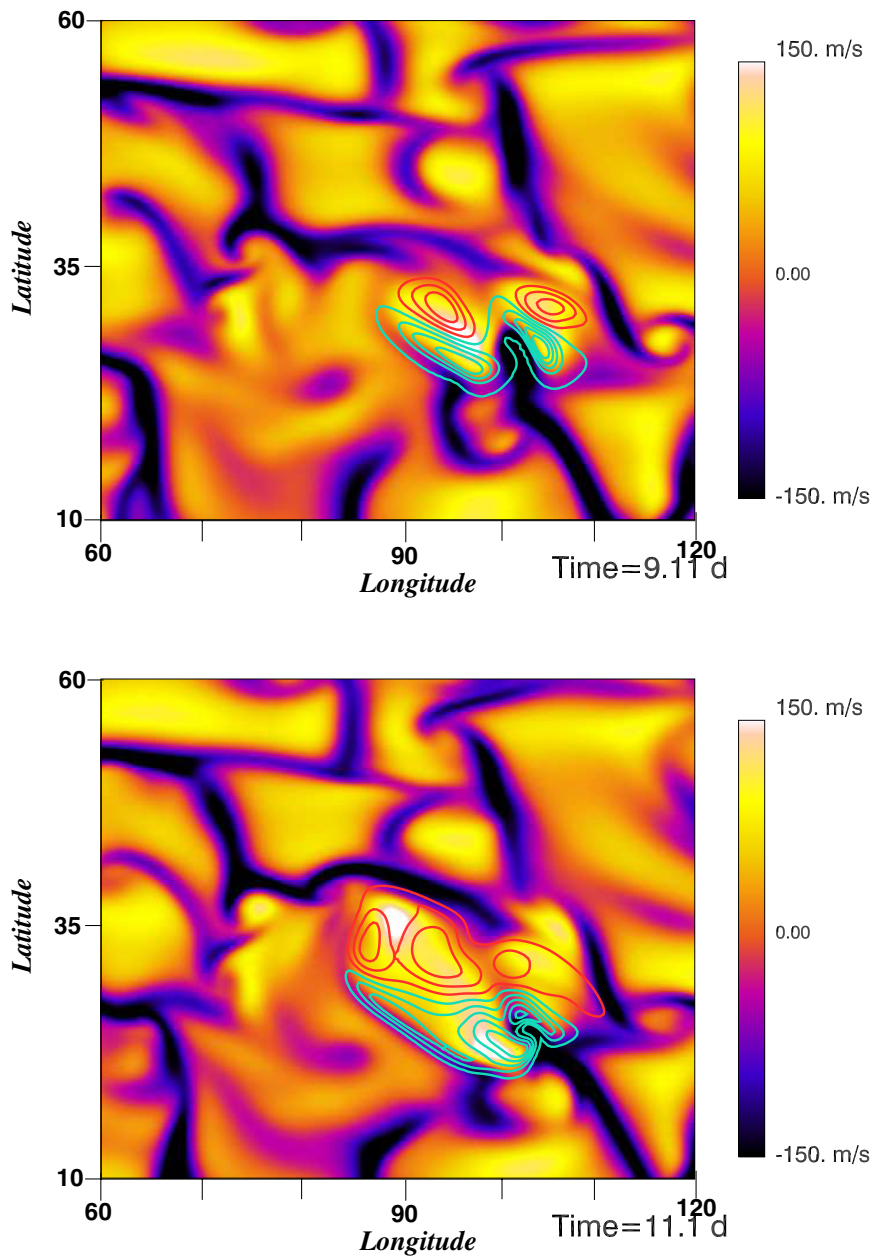

Case 4TwP
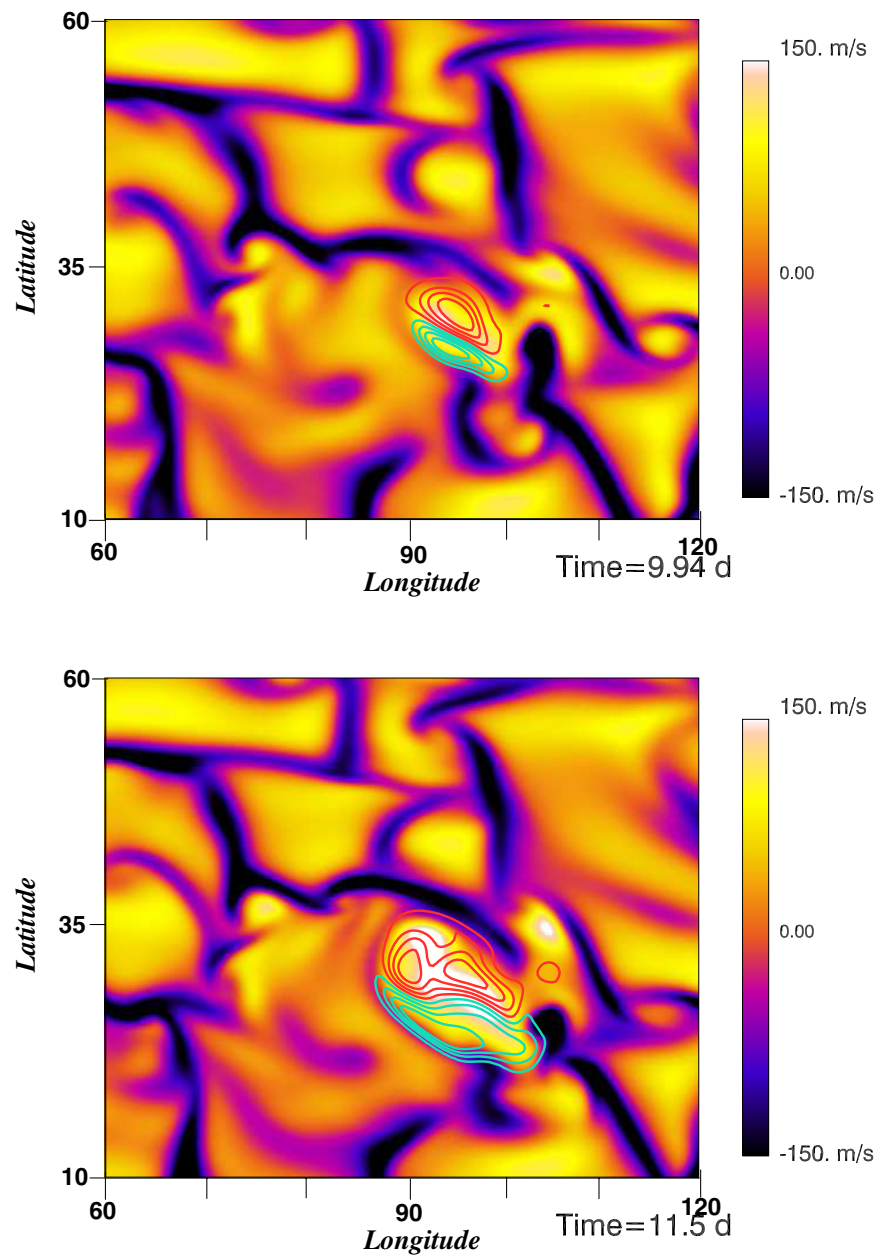

Figure 17. Same as Figure 16, but for Cases 2TwP and 4TwP. The typical magnetic field strengths here are between $5000 \mathrm{G}$ and $8000 \mathrm{G}$.

(A color version of this figure is available in the online journal.)

$500 \mathrm{G}$ in the convective cases. This is related to the explanations of Section 5.1 where the buoyancy loss was shown and thought to be partially due to the convective motions creating smaller scales and thus stronger dissipation.

Another striking feature is the strong increase of negative $B_{r}$ in Case 1TwP (panel (a)) at $t=13$ days and of positive $B_{r}$ in Case 1TwN (panel (b)) at about the same time. This is mainly due to the fact that these cases are dominated by convective motions. Indeed, as we saw in Figure 16, the negative polarity in Case 1TwP between $t=12$ and $t=16$ days is strongly squeezed by the convective motions in the narrow downflow lanes. This compression of the magnetic field tends to produce a stronger concentration of fields, explaining the strong increase of negative $B_{r}$ in Case 1TwP and then its sharp decrease due to fast dissipation of small scales. A similar situation happens in Case 1TwN for the opposite dominating polarity. In Cases 3TwP and 3TwN (panels (c) and (d)), where the initial magnetic field is twice as large, such sharp increase and decrease of the two polarities are not visible since the loop emerges as a global magnetic structure and is much less influenced by convective motions. It is worth noting that we may have a possible way here to explain the formation of complex active regions from where powerful (X-class) flares could originate. Indeed, active regions with a complex topology could be created by the turbulent convective motions acting on moderately strong flux tubes (of approximately $5 \times 10^{4} \mathrm{G}$ at the base of the $\mathrm{CZ}$ ). By the time they reach the top of the convection zone, the flux tubes have been strongly deformed and the subsequent emerging radial field consists of several patches of opposite polarities, as seen in Figure 16. We could argue that this complex active region, whose progenitor is a relatively weak flux tube, would not be able to produce very energetic flares. However, when panel (a) of Figure 18 is considered, we conclude that the converging motions at the sub-photospheric level are able to re-concentrate the magnetic structure in the strong downdrafts, in order to locally produce a strong increase of the radial field. In particular, we note that the maximum positive radial field is about twice as large as the negative $B_{r}$, which was not the case in the isentropic case where the re-concentration by convective motions could not occur. This scenario may thus explain the injection of energy in the corona by convective motions acting on complex active regions originating from initially weak flux tubes.

As far as the tilt angle is concerned in these simulations, the influence of the initial twist, which is not included in the thin flux tube calculations, is again crucial. If we follow the position in latitude of the positive trailing and negative leading polarities (not shown here), then we find that at the beginning of emergence, the leading polarity is located at higher latitude than the leading one, unlike in solar observations. However, in Case 1 TwN, the clockwise rotation due to the Coriolis force 

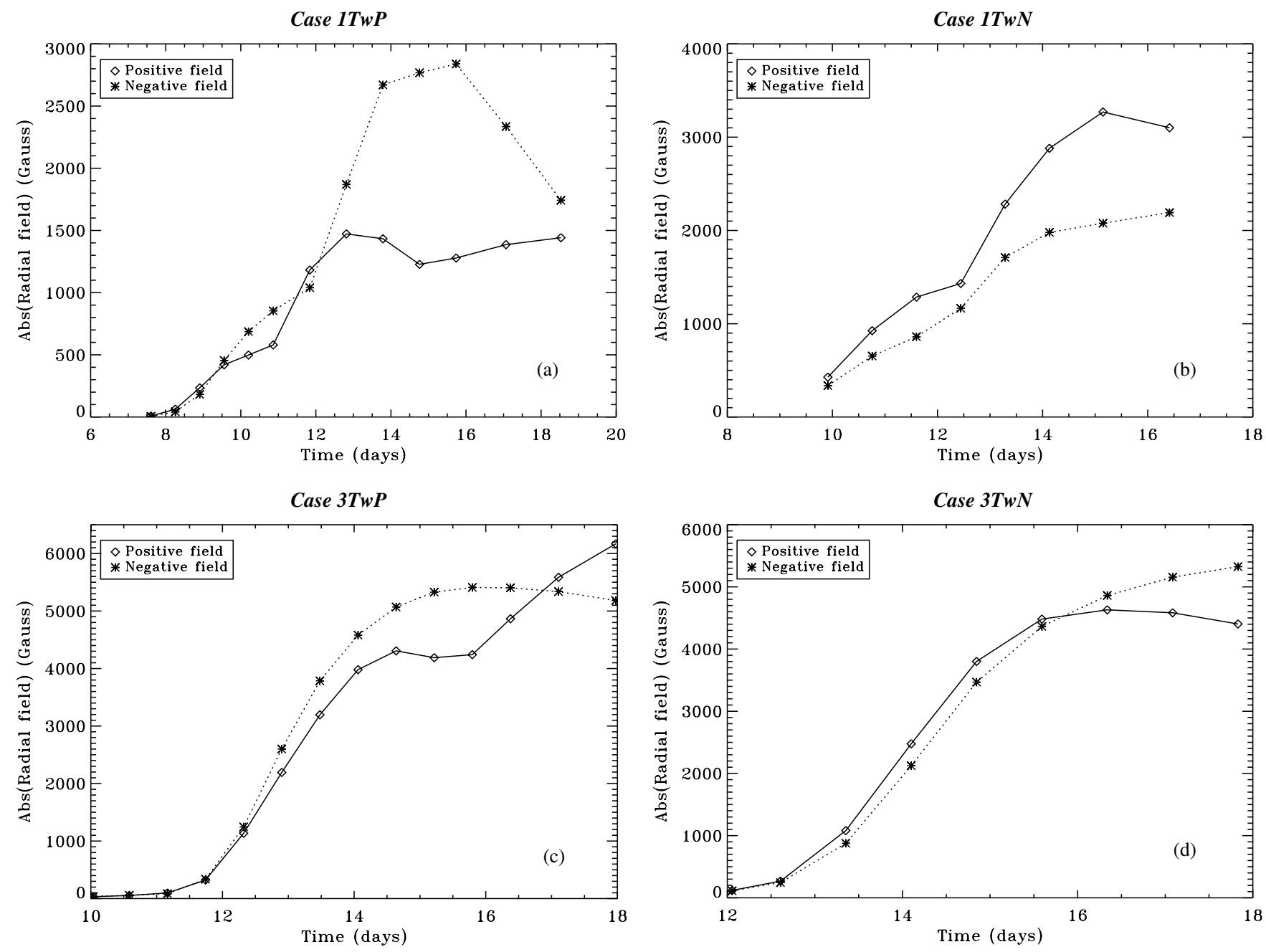

Figure 18. Temporal evolution of the radial magnetic field intensity in the trailing and leading polarities at and right after emergence, for cases 1TwP (panel (a)), 1 TwN (panel (b)), 3TwP (panel (c)), and 3TwN (panel (d)).

acting on the legs of the loop tends to reverse this situation about five days after emergence, implying a leading polarity at slightly lower latitude than the trailing one and a tilt of about $5^{\circ}$, in agreement with Joy's law. This is similar to what was found in the isentropic Case 1TwN. Case 3TwN does not follow the same evolution. Indeed, the orientation at emergence is kept even after 10 days after emergence before the magnetic field is swept toward the downflow lanes and dissipates. Again, we need a loop that is sufficiently influenced by the Coriolis force to get a tilt in agreement with observations with an initial left-handed twist believed to dominate in the Northern Hemisphere.

Of course, not only will the initial twist play a role in the tilt of the emerging region but also the latitude of introduction and emergence, as already pointed out in the isentropic cases. We thus computed extra cases as in the isentropic simulations, Cases 2TwP15 and 2TwP60. We did not choose the same initial parameters as in the isentropic cases to study the effect of latitude, since Case 1 was computed in the isentropic case and it is believed here to be influenced too much by convection to produce a significant trend, and thus to allow us to draw any conclusions on the tilt at the time of emergence at $0.93 R_{\odot}$. Case 2TwP is the most interesting, since it is still influenced by convection (as we saw in the previous section) without completely loosing its characteristic bipolar structure when the loop emerges at the top of our domain. The results of these extra calculations are shown in Figure 19. Both the emerging radial magnetic field and a measure of the tilt angle are represented, similar to Figure 10. We argue that some effects of the differential rotation are visible here. First, the rise velocities are different from one case to another, like the isentropic simulations, but the differences are even more pronounced here. To be more precise, the characteristic rise time for the loop initially located at a latitude of $60^{\circ}$ is around 8 days, while it is of more than 11 days for the case at latitude $=15^{\circ}$. The figures represent the emerging region about 1 to 2 days after the first signs of emergence at $r=0.93 R_{\odot}$. This slow down of the loops located at lower latitudes is related to the rotation rate, which is stronger at lower latitudes. Indeed, as shown already in Jouve \& Brun (2007), the modified buoyancy force in the presence of rotation is proportional to $\Delta \rho\left(g-r \sin ^{2} \theta \Omega^{2}\right)$ and the rise velocity of the loop will thus be less at low latitudes and high rotation rates, which is typically the situation for Case 2TwP15, represented here in the first panel of Figure 19. A second notable effect of the differential rotation concerns the longitude of emergence. In these differentially rotating cases, the loop which was introduced at higher latitudes has drifted in longitude by about $30^{\circ}$ (it was introduced at $100^{\circ}$ and emerges at $70^{\circ}$ ), while the loop in Case2TwP15 has only drifted by $5^{\circ}$. This is quite different from what we had in the isentropic cases, where the drift in longitude was more 


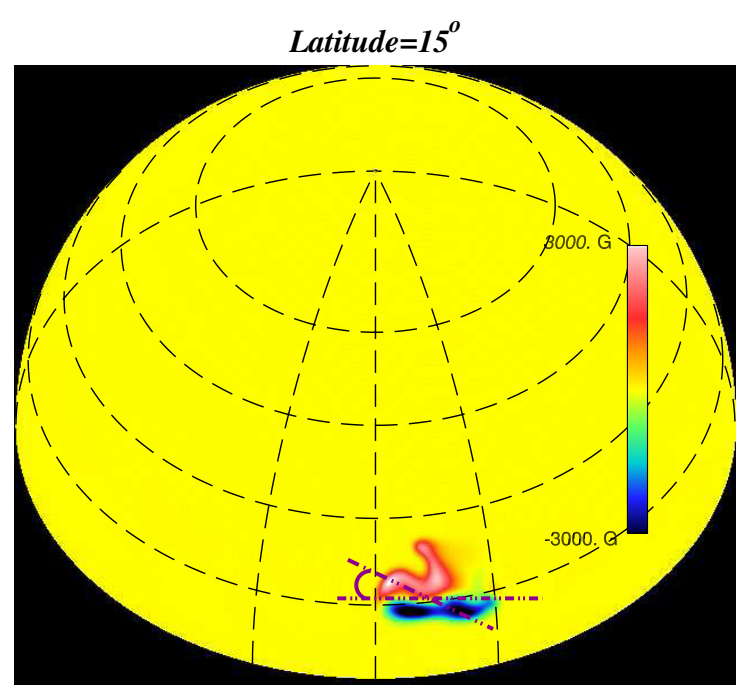

Time $=12.5 \mathrm{~d}$

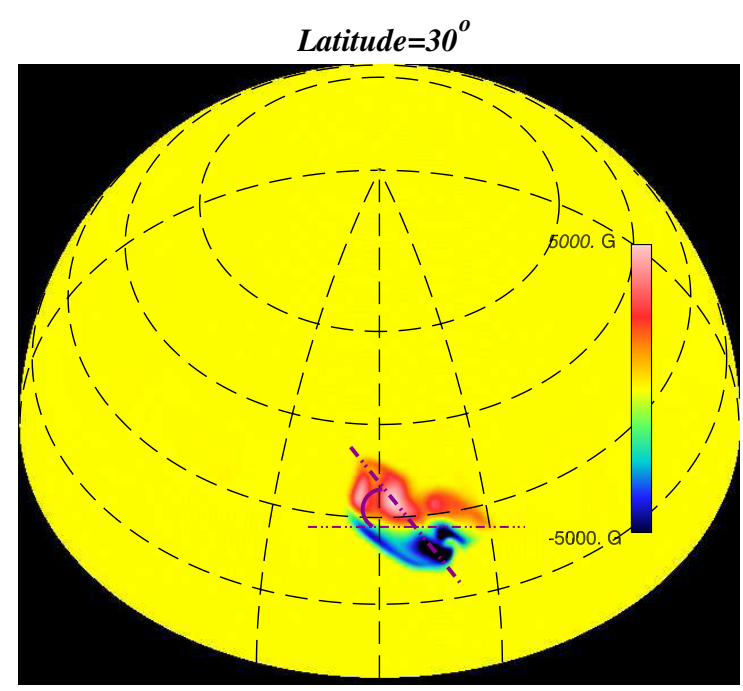

Time=11.07 d

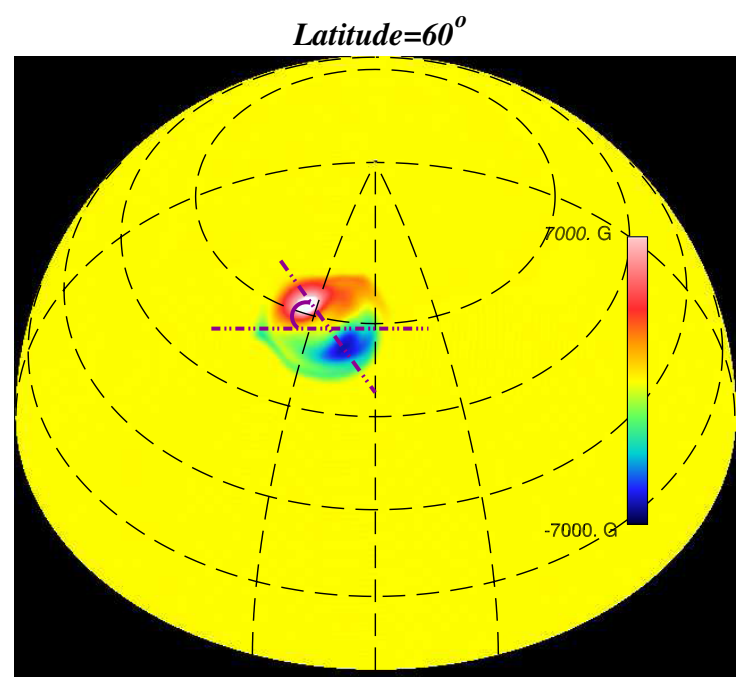

Time=10.2 d

Figure 19. Same as Figure 10, but for the convective Cases 2TwP15, 2TwP, and 2TwP60.

(A color version of this figure is available in the online journal.) important for the case at low latitudes. This was due to the stronger asymmetry between the two legs of the loop which appeared in the low latitude case. Here, again, the different drift values could very well be due to the differential rotation. Indeed, since the higher (lower) latitudes rotate retrograde (prograde) compared to the rotating frame, it is not surprising that the loop at high latitudes will be globally shifted at lower longitudes compared to the low latitude cases. This is thus a clear effect of the large-scale differential rotation acting on our rising $\Omega$-loops. Finally, the idea here was to compare the tilt angles we could get in these convective cases and then compare those values to observations. It is more complicated here to identify well-defined concentrations of opposite polarities, since the convective motions have influenced the loops during their rise to sometimes produce several concentrations of radial magnetic field of both polarities. However, if we consider that the tilt angle is defined as the angle between the East-West direction and the line joining the maximum of both polarities, then we are still able to measure a tilt in those three cases. We find a value of about $20^{\circ}$ for Case2TwP15, $40^{\circ}$ for Case2TwP and $45^{\circ}$ for Case2TwP60. The trend is thus still to increase with increasing latitude but it is less obvious than in the isentropic cases. We note here that the tilt angle distribution in the simulations of Weber et al. (2011) had much more of a spread in the convective cases than in the isentropic ones, which is consistent with our simulations. This is an indication that it is not only the rotation and the associated Coriolis force acting on the loop that will determine the tilt angle observed at the solar surface, but horizontal buffeting by convection could also strongly modify this measurement, even if the trend compatible with Joy's law is still found in those cases.

\section{DISCUSSION AND CONCLUSIONS}

In this work, we presented the dynamical evolution of twisted $\Omega$-loops embedded in a convectively stable and unstable unmagnetized environment to mimic the behavior of a strong toroidal magnetic field generated by dynamo action at the base of the solar convection zone and rising toward the photosphere. Those simulations allow us to take into account the full effects of sphericity, mean flows, and convective motions on the dynamical evolution of a flux rope with a finite radius, and thus contain much more physics than previous thin flux tube calculations. The first part of this work was dedicated to establishing the important parameters to obtain a coherent radial rise in the isentropic case. We find that a loop with an initial magnetic field of $5 \times 10^{4} \mathrm{G}$ and with an initial twist of less than one turn along the loop extension ( $15^{\circ}$ here) is able to rise and emerge at the top of our domain to create bipolar regions. Asymmetries develop between the leading and trailing legs of the loop due to the combined effect of angular momentum conservation and Lorentz forces, and it is found that this asymmetry is strongly reduced when magnetic structures as strong as $1.5 \times 10^{5} \mathrm{G}$ are considered. The emerging regions obtained in these isentropic cases have several properties that agree with observations. In particular, well-identified tongues develop during emergence, mainly due to the initial twist of the magnetic field lines, also seen in observations (e.g., Lopez Fuentes et al. 2000). For the first time, we point out the appearance during flux emergence of sharp ring-shaped magnetic structures around the bipolar region that we call "magnetic necklaces" and which are due to the creation of vorticity at the loop periphery. They are also very often seen in observations of large-scale flux emergence (especially in Liu \& Zhang 2006 with MDI data) and are very 
similar in shape to the ones we obtain in our simulated active regions. Moreover, we show here that it is possible to get a case where the initial left-handed twist is strong enough to maintain the coherence of the loop and where the tilt angle of the emerging radial field agrees with Joy's law (in contrast to what is found in Fan 2008). To do so, the rise time needs to be long enough so that the Coriolis force acts sufficiently on the loop to rotate the two opposite polarities clockwise and then produce a final tilt angle of the correct sign.

We note that if much thinner flux tubes were considered, then the various parameters quoted here to get bipolar regions with properties in agreement with observations would probably have to be revised. The results do not change significantly if a tube four times smaller is introduced, as long as the magnetic diffusivity is also reduced to keep the rise time small compared to the diffusion time of the magnetic structure (see Jouve \& Brun 2009 , for a discussion on the effect of diffusion on this type of simulations). However, if much thinner tubes are introduced (say 10 to 20 times smaller, as can be expected in the real Sun), then it is likely that the results may differ. In particular, the drag force coming from the pressure difference between the upand downstream sides of the turbulent wake behind the rising loop may be much bigger when the loop radius is reduced. This was shown in Batchelor (1967) for incompressible flows passing a rigid cylinder under large Reynolds number conditions and shown to be still true for two-dimensional simulations of buoyant loops (Emonet \& Moreno-Insertis 1998). This will in turn modify the rise velocity and the competition between magnetic fields and convective motions. For example, a smaller rise velocity leaves more time for the Coriolis force to act, but at the same time, an increased drag directly opposes the tilting motion, resulting in a net decrease of the tilt angle of loops of smaller radii, as shown by D'silva \& Choudhuri (1993). This work would then benefit from studying the effect of loops of smaller radii but this question was not addressed here. Pinto \& Brun (2012) also find that the wake structure is modified by the presence of a background dynamo field, possibly due to continuous field reconnection as the tube rises.

In the convective cases, the rise velocity and characteristics of emerging regions are strongly affected by the convective motions when loops of less than $10^{5} \mathrm{G}$ are considered. However, emerging regions with the correct orientation and a dominant leading polarity are still found in these simulations where the full effects of convection, rotation, and sphericity are taken into account. Making direct comparison with observed photospheric field intensity in active regions is not straightforward since the scaling of the magnetic field strength with density (which is linear in our cases) may change drastically when reaching the uppermost layers of the convection zone (Cheung et al. 2010). However, local helioseismology techniques seem to start to provide us with constraints on the field intensity deeper down (Ilonidis et al. 2011, 2012; Braun 2012), much closer to the top of our computational domain. We may well soon be able to much more directly relate the results of our three-dimensional simulations to actual observations.

If the origin of the tilt angle of active regions has not been completely related to one particular property of the emerging loops yet, then it is undeniable that the initial twist of the field lines is an important factor. It is thus necessary to understand the generation not only of buoyant magnetic field at the base of the convection zone but of twisted buoyant structures. It is believed that such twisted loops could be created by the advection of poloidal field lines by the initially rising strong toroidal field subject to magnetic buoyancy instabilities (Longcope et al. 1996; Favier et al. 2012). Full MHD simulations of the dynamogenerated toroidal field becoming buoyant, gaining some twist, and being able to rise coherently to the top of the convection zone are in progress (Nelson et al. 2011, 2012). We moreover note that such simulations are able to mimic the dynamical evolution of a magnetic structure from the base of the convection zone to about $30 \mathrm{Mm}$ under the photosphere and that emergence higher up is not possible in these global anelastic simulations. Contact with three-dimensional compressible local simulations of emerging flux in the solar atmosphere (Archontis \& Hood 2010) should now be considered to have a full picture of the MHD evolution of large-scale magnetic regions.

When convection is considered, the emerging radial field may lose the typical characteristics of a well-defined bipolar structure that we get in the isentropic cases. Indeed, in Case 1TwP, where the evolution was dominated by the downflows and upflows of the surrounding convection, the emerging structure is very complex and regions of mixed polarity are self-consistently formed. The large variety of large-scale emerging regions at the solar photosphere may then well be the results of magnetic $\Omega$-loops significantly processed by convection, even if robust statistical properties such as tilt angle or asymmetry between polarities indicate that these loops are still able to keep their coherence during their rise. Another possibility leading to the emergence of complex active regions in the Sun could be that such buoyant structures might interact in the bulk of the convection zone. Depending on which kind of reconnection could occur between the $\Omega$-loops, either a complex region with mixed polarity and mixed helicity could emerge (such as the one observed by Chandra et al. 2010), or the magnetic field could completely change its trajectory, leading to no emergence at all (see Linton \& Antiochos 2005 for a discussion of reconnection between twisted flux tubes). This is the analysis we intend to focus on in the near future. Furthermore, no background dynamo field was present in the simulation. Pinto \& Brun (2012) have started to address the influence of such a dynamo field on a uniformly buoyant magnetic rope. We intend to carry on this analysis for $\Omega$-loops.

We thank the organizers of the Flux Emergence Workshops held in St. Andrews in 2007 June, in Kyoto in 2008 October, and in Berkeley in 2011 August. We also acknowledge financial support by the ERC through grant 207430 STARS2 and funding by the Programme National Soleil Terre (INSU/PNST). We also appreciate the GENCI supercomputing centers for granting access to their infrastructures through project 1623.

\section{REFERENCES}

Abbett, W. P., Fisher, G. H., \& Fan, Y. 2000, ApJ, 540, 548

Acheson, D. J. 1979, SoPh, 62, 23

Archontis, V., \& Hood, A. W. 2010, A\&A, 514, 56

Archontis, V., \& Török, T. 2008, A\&A, 492, 35

Aulanier, G., Török, T., Démoulin, P., \& DeLuca, E. E. 2010, ApJ, 708, 314

Batchelor, G. K. 1967, An Introduction to Fluid Dynamics (Cambridge: Cambridge Univ. Press)

Braun, D. C. 2012, Sci., 336, 296

Browning, M. K., Miesch, M. S., Brun, A. S., \& Toomre, J. 2006, ApJ, 648, L157

Brun, A. S. 2004, SoPh, 220, 333

Brun, A. S., Antia, H. M., Chitre, S. M., \& Zahn, J.-P. 2002, A\&A, 391, 725

Brun, A. S., Miesch, S. M., \& Toomre, J. 2004, ApJ, 614, 1073

Caligari, P., Moreno-Insertis, F., \& Schüssler, M. 1995, ApJ, 441, 886

Caligari, P., Schüssler, M., \& Moreno-Insertis, F. 1998, ApJ, 502, 481

Cattaneo, F., \& Hughes, D. W. 1988, JFM, 196, 323 
Chae, J., \& Moon, Y.-J. 2005, ApJ, 629, 1110

Chandra, R., Pariat, E., Schmieder, B., Mandrini, C. H., \& Uddin, W. 2010, SoPh, 261,127

Charbonneau, P. 2005, LRSP, 2, 2

Cheung, M. C. M., Rempel, M., Title, A. M., \& Schüssler, M. 2010, ApJ, 720,233

Choudhuri, A., \& Gilman, P. 1987, ApJ, 316, 788

Clune, T. L., Elliott, J. R., Glatzmaier, G. A., Miesch, M. S., \& Toomre, J. 1999, ParC, 25, 361

Démoulin, P., Mandrini, C. H., van Driel-Gesztelyi, L., et al. 2002, A\&A, 382,650

D'silva, S., \& Choudhuri, A. R. 1993, A\&A, 272, 621

Emonet, T., \& Moreno-Insertis, F. 1998, ApJ, 492, 804

Fan, Y. 2001, ApJ, 554, 111

Fan, Y. 2004, LRSP, 1, 1

Fan, Y. 2008, ApJ, 676, 680

Fan, Y., Abbett, W. P., \& Fisher, G. H. 2003, ApJ, 582, 1206

Fan, Y., Fischer, G. H., \& DeLuca, E. E. 1993, ApJ, 405, 390

Fan, Y., \& Gibson, S. E. 2004, ApJ, 609, 1123

Favier, B., Jouve, L., Edmunds, W., Silvers, L. J., \& Proctor, M. R. E. 2012, MNRAS, 426, 3349

Gilman, P. A., \& Glatzmaier, G. A. 1981, ApJS, 45, 335

Hale, G. E., Ellerman, F., Nicholson, S. B., \& Joy, A. H. 1919, ApJ, 49, 153

Ilonidis, S., Zhao, J., \& Kosovichev, A. 2011, Sci, 333, 993

Ilonidis, S., Zhao, J., \& Kosovichev, A. 2012, Sci, 336, 296

Jouve, L., \& Brun, A. S. 2007, Astron. Notes, 328, 1104

Jouve, L., \& Brun, A. S. 2009, ApJ, 701, 1300

Kosovichev, A. G., \& Stenflo, J. O. 2008, ApJ, 688, L115

Leka, K. D., Canfield, R. C., McClymont, A. N., \& van Driel-Gesztelyi, L. 1996, ApJ, 462, D547

Linton, M., \& Antiochos, S. K. 2005, ApJ, 625, 506

Liu, J., \& Zhang, H. 2006, SoPh, 234, 21

Longcope, D. W., Fisher, G. H., \& Arendt, S. 1996, ApJ, 464, 999

Lopez Fuentes, M. C., Démoulin, P., Mandrini, C. H., \& van Driel-Gesztelyi, L. 2000, ApJ, 544, 540

Luoni, M. L., Démoulin, P., Mandrini, C. H., \& van Driel-Gesztelyi, L. 2011, SoPh, 270, 45

Magara, T. 2004, ApJ, 605, 480

Manchester, W., IV, Gombosi, T., DeZeeuw, D., \& Fan, Y. 2004, ApJ, 610,588

Martínez-Sykora, J., Hansteen, V., \& Carlsson, M. 2008, ApJ, 679, 871

Matthews, P. C., Hughes, D. W., \& Proctor, M. R. E. 1995, ApJ, 448, 938
Miesch, M. S. 2005, LRSP, 2, 1

Miesch, M. S., Brun, A. S., \& Toomre, J. 2006, ApJ, 641, 618

Miesch, M. S., Elliott, J. R., Toomre, J., et al. 2000, ApJ, 532, 593

Moffatt, H. K. 1978, Magnetic Field Generation in Electrically Conducting Fluids (Cambridge: Cambridge Univ. Press), 353

Moreno-Insertis, F., Schüssler, M., \& Caligari, P. 1994, SoPh, 153, 449

Moreno-Insertis, F., Schüssler, M., \& Ferriz Mas, A. 1992, A\&A, 264, 686

Murray, M. J., Hood, A. W., Moreno-Insertis, F., Galsgaard, K., \& Archontis, V. 2006, A\&A, 460, 909

Nelson, N. J., Brown, B. P., Brun, A. S., Miesch, M. S., \& Toomre, J. 2011, ApJ, 739,38

Nelson, N. J., Brown, B. P., Brun, A. S., Miesch, M. S., \& Toomre, J. 2012, ApJ, accepted

Ossendrijver, M. 2003, A\&AR, 11, 287

Otsuji, K., Kitai, R., Ichimoto, K., \& Shibata, K. 2011, PASJ, 63, 1047

Parker, E. N. 1955, ApJ, 122, 293

Parker, E. N. 1993, ApJ, 408, 707

Pariat, E., Aulanier, G., Schmieder, B., et al. 2004, ApJ, 614, 1099

Pinto, R., \& Brun, A. S. 2012, ApJ, submitted

Pomarède, D., \& Brun, A. S. 2010, in ASP Conf. Ser. 434, Astronomical Data Analysis Software and Systems XIX, ed. Y. Mizumoto, K.-I. Morita, \& M. Ohishi (San Francisco, CA: ASP), 378

Sanchez Almeida, J. 1998, in ASP Conf. Ser. 155, Three Dimensional Structure of Solar Active Regions, ed. C. E. Alissandrakis \& B. Schmieder (San Francisco, CA: ASP), 54

Schrijver, C. J., \& Harvey, K. L. 1994, SoPh, 150, 1

Schüssler, M., Caligari, P., Ferriz-Mas, A., \& Moreno-Insertis, F. 1994, A\&A, 281,69

Spruit, H. C. 1981, A\&A, 98, 155

Török, T., \& Kliem, B. 2005, ApJ, 630, L97

van Driel-Gesztelyi, L. 2002, in Proc. Magnetic Coupling of the Solar Atmosphere Euroconference, SOLMAG 2002, ed. H. Sawaya-Lacoste (ESA SP-505; Noordwijk: ESA), 113

van Driel-Gesztelyi, L., Démoulin, P., Mandrini, C. H., Harra, L., \& Klimchuk, J. A. 2003, ApJ, 586, 579

Wang, Y.-M., \& Sheeley, N. R., Jr. 1989, SoPh, 124, 81

Wang, Y.-M., \& Sheeley, N. R., Jr. 1991, ApJ, 375, 761

Weber, M. A., Fan, Y., \& Miesch, M. S. 2011, ApJ, 741, 11

Weiss, N. O., Brownjohn, D. P., Matthews, P. C., \& Proctor, M. R. E. 1996, MNRAS, 283, 1153

Wong, V. C., \& Lilly, D. K. 1994, PhFl, 6, 1016

Zwann, C. 1987, ARA\&A, 25, 83 